\title{
Final characterisation and design of the Gamma-ray Cherenkov Telescope (GCT) for the Cherenkov Telescope Array
}

O. Le Blanc, G. Fasola, J. M. Huet, R. White, A. Dmytriiev, et al.

O. Le Blanc, G. Fasola, J. M. Huet, R. White, A. Dmytriiev, H. Sol, A. Zech, A. Abchiche, J. P. Amans, T. P. Armstrong, M. Barcelo, D. Berge, A. M. Brown, G. Buchholtz, P. M. Chadwick, P. Clark, G. Cotter, L. Dangeon, F. De Frondat, P. Deiml, J. L. Dournaux, C. Duffy, S. Einecke, S. Flis, S. Funk, G. Giavitto, J. Gironnet, J. A. Graham, T. Greenshaw, J. A. Hinton, I. Jégouzo, M. Kraus, J. S. Lapington, P. Laporte, S. A. Leach, S. Lloyd, I. A. Minaya, R. Morier, A. Okumura, H. Prokoph, D. Ross, G. Rowell, C. B. Rulten, H. Schoorlemmer, J. Schmoll, S. T. Spencer, M. Stephan, R. Stuik, H. Tajima, J. Thornhill, L. Tibaldo, J. Vink, J. J. Watson, J. Williams, A. Zink, J. Zorn, "Final characterisation and design of the Gamma-ray Cherenkov Telescope (GCT) for the Cherenkov Telescope Array," Proc. SPIE 10700, Ground-based and Airborne Telescopes VII, 1070010 (6 July 2018); doi: $10.1117 / 12.2313158$

Event: SPIE Astronomical Telescopes + Instrumentation, 2018, Austin, Texas, United States 


\title{
Final characterisation and design of the Gamma-ray Cherenkov Telescope (GCT) for the Cherenkov Telescope Array
}

\author{
O. Le Blanc*a, G. Fasola ${ }^{\mathrm{a}}$, J.M. Huet ${ }^{\mathrm{a}}$, R. White ${ }^{\mathrm{b}}$, A. Dmytriiev ${ }^{\mathrm{a}}$, H. Sol ${ }^{\mathrm{a}}$, A. Zech ${ }^{\mathrm{a}}$, A. Abchiche ${ }^{\mathrm{c}}$, \\ J.P. Amans ${ }^{\mathrm{a}}$, T.P. Armstrong ${ }^{\mathrm{d}}$, M. Barcelo ${ }^{\mathrm{b}}$, D. Berge ${ }^{\mathrm{e}}$, A.M. Brown ${ }^{\mathrm{f}}$, G. Buchholtz ${ }^{\mathrm{c}}$, \\ P.M. Chadwick ${ }^{\mathrm{f}}$, P. Clark ${ }^{\mathrm{f}}$, G. Cotter ${ }^{\mathrm{d}}$, L. Dangeon ${ }^{\mathrm{a}}$, F. De Frondat ${ }^{\mathrm{a}}$, P. Deiml ${ }^{\mathrm{g}}$, J.L. Dournaux ${ }^{\mathrm{a}}$, \\ C. Duffy ${ }^{\mathrm{h}}$, S. Einecke, , S. Flis ${ }^{\mathrm{e}}$, S. Funk ${ }^{\mathrm{f}}$, G. Giavitto ${ }^{\mathrm{e}}$, J. Gironnet ${ }^{\mathrm{c}}$, J.A. Graham ${ }^{\mathrm{f}}$, T. Greenshaw ${ }^{\mathrm{j}}$, \\ J.A. Hinton ${ }^{\text {b }}$ I. Jégouzo ${ }^{\mathrm{a}}$, M. Kraus ${ }^{\text {, }}$, J.S. Lapington ${ }^{\mathrm{h}}$, P. Laporte ${ }^{\mathrm{a}}$, S.A. Leach ${ }^{\mathrm{h}}$, S. Lloyd ${ }^{\mathrm{f}}$, \\ I.A. Minaya ${ }^{\mathrm{j}}$, R. Morier ${ }^{\mathrm{a}}$, A. Okumura ${ }^{\mathrm{k}}$, H. Prokoph ${ }^{\mathrm{e}}$, D. Ross ${ }^{\mathrm{h}}$, G. Rowelli, C.B. Rulten , \\ H. Schoorlemmer ${ }^{\mathrm{b}}$, J. Schmoll , S.T. Spencer ${ }^{\mathrm{d}}$, M. Stephan ${ }^{1}$, R. Stuik ${ }^{\mathrm{m}}$, H. Tajimak ${ }^{\mathrm{k}}$, J. Thornhill ${ }^{\mathrm{h}}$, \\ L. Tibaldo ${ }^{\mathrm{n}}$, J. Vink $\mathrm{V}^{1}$, J.J. Watson ${ }^{\mathrm{d}}$, J. Williams ${ }^{\mathrm{h}}$, A. Zink ${ }^{\mathrm{g}}$, J. Zorn ${ }^{\mathrm{b}}$ for the CTA GCT Project
}

${ }^{a}$ Observatoire de Paris, CNRS, PSL Research University, LUTH \& GEPI, 5 Place J. Janssen, 92195,

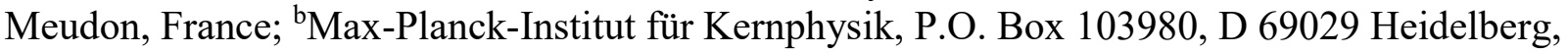
Germany; ${ }^{\circ}$ CNRS, Division technique DT-INSU, 1 Place Aristide Briand, 92190 Meudon, France;

${ }^{\mathrm{d}}$ Department of Physics, University of Oxford, Keble Road, Oxford OX1 3RH, UK; ${ }^{\mathrm{e}}$ Deutsches Elektronen-Synchrotron, Platanenallee 6, D-15738 Zeuthen, Germany; ${ }^{\mathrm{f} D e p a r t m e n t ~ o f ~ P h y s i c s ~ a n d ~}$ Centre for Advanced Instrumentation, Durham University, South Road, Durham DH1 3LE, UK; ${ }^{g}$ Erlangen Centre for Astroparticle Physics, Erwin- Rommel-Str. 1, D 91058 Erlangen, Germany;

${ }^{\mathrm{h}}$ Department of Physics and Astronomy, University of Leicester, University Road, Leicester, LE1 7RH, UK; i School of Physical Sciences, University of Adelaide, Adelaide5005, Australia; ${ }^{j}$ University of Liverpool, Oliver Lodge Laboratory, P.O. Box 147, Oxford Street, Liverpool L69 3BX, UK; ${ }^{k}$ Institute for Space-Earth Environmental Research, Nagoya University, Furo-cho, Chikusa-ku, Nagoya, Aichi 464-8601, Japan; 'Gravitation and AstroParticle Physics in Amsterdam,

University of Amsterdam, Science Park 904, 1098 XH Amsterdam, The Netherlands; ${ }^{\mathrm{m}}$ Leiden Observatory, Leiden University, Postbus 9513, 2300 RA, Leiden, Netherlands; ' Institut de recherche en Astrophysique et Planétologie, 9 Avenue du Colonel Roche, 31028 Toulouse, France

\begin{abstract}
The Gamma-ray Cherenkov Telescope (GCT) is one of the telescopes proposed for the Small Sized Telescope (SST) section of CTA. Based on a dual-mirror Schwarzschild-Couder design, which allows for more compact telescopes and cameras than the usual single-mirror designs, it will be equipped with a Compact High-Energy Camera (CHEC) based on silicon photomultipliers (SiPM). In 2015, the GCT prototype was the first dual-mirror telescope constructed in the prospect of CTA to record Cherenkov light on the night sky. Further tests and observations have been performed since then. This report describes the current status of the GCT, the results of tests performed to demonstrate its compliance with CTA requirements, and the optimisation of the design for mass production. The GCT collaboration, including teams from Australia, France, Germany, Japan, the Netherlands and the United Kingdom, plans to install the first telescopes on site in Chile for 2019-2020 as part of the CTA pre-production phase.
\end{abstract}

Keywords: Imaging Atmospheric Cherenkov Telescopes, CTA, GCT, Schwarzschild-Couder telescope, Telescope Control System, Aspherical mirrors, SiPM detector, Full-waveform read-out

*oriane.le-blanc@obspm.fr 


\section{INTRODUCTION}

The Cherenkov Telescope Array (CTA) project $[1]$ aims to build the next generation ground-based Very High-Energy (VHE) instrument after HESS, MAGIC and VERITAS. It will be devoted to the observation of gamma rays over a large band of energy from a few tens of $\mathrm{GeV}$ up to $300 \mathrm{TeV}$ and will be ten times more sensitive than current Imaging Atmospheric Cherenkov Telescopes (IACTs) in the multi-TeV domain as shown in Figure 1. All other performances such as angular, spectral and time resolution will also be significantly improved and several observing modes will be available. Two sites are foreseen in the Southern (close to Paranal Observatory, Chile) and Northern (Observatorio del Roque de los Muchachos in La Palma, Canary Islands) hemispheres to cover the whole sky. The southern CTA array will host IACTs of three different sizes: 4 Large-Sized Telescopes (LSTs - mirror diameter $23 \mathrm{~m}$ ), 25 Medium-Sized Telescopes (MSTs $12 \mathrm{~m}$ ) and 70 Small-Sized Telescopes (SSTs $-4 \mathrm{~m}$ ). The latter will open a new electromagnetic window at extreme energies, above a few TeV and up to $300 \mathrm{TeV}$, making it possible to search for PeVatrons in our Galaxy and in the Large Magellanic Cloud, to study hadronic and leptonic emissions from nearby Active Galactic Nuclei at extreme energies, and to explore topics in cosmology and fundamental physics, such as Dark Matter, Extragalactic Background Light, Intergalactic Magnetic Field, Lorentz Invariance Violation and Axion-Like Particles.

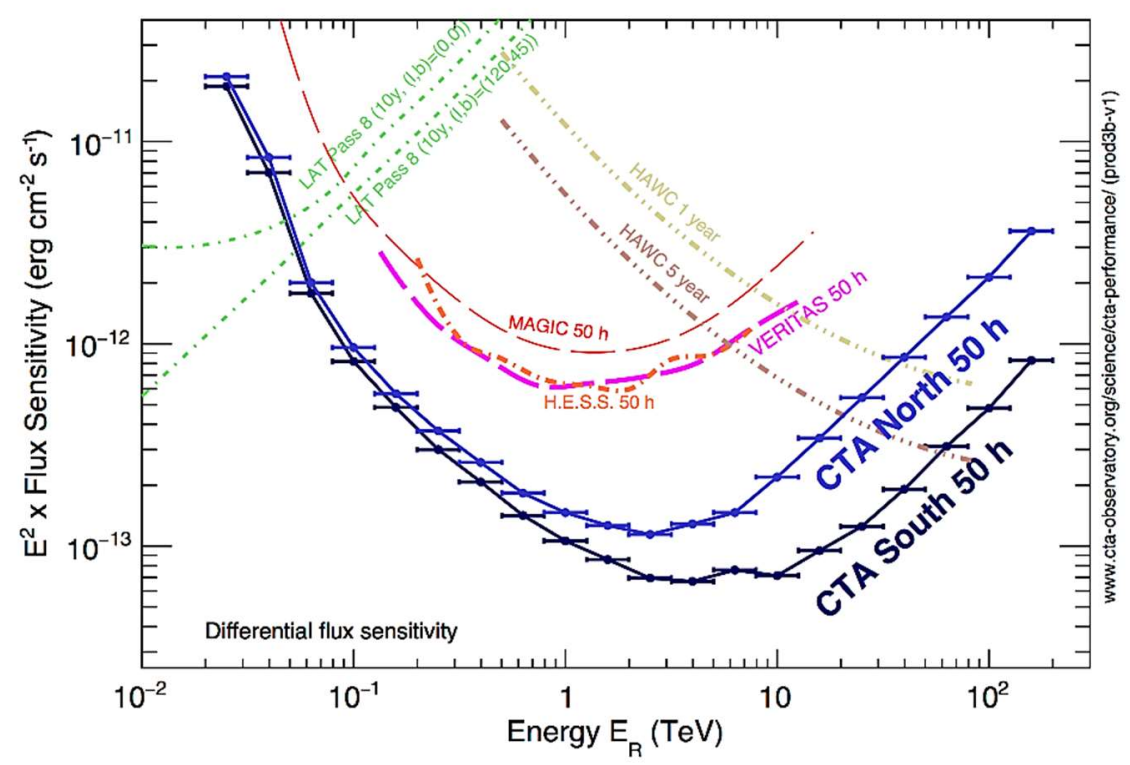

Figure 1. The differential flux sensitivity of CTA North and South compared to the current performance of existing IACT systems, Fermi-LAT and HAWC.

The Gamma-ray Cherenkov Telescope (GCT) is one of the telescopes ${ }^{[2]}$ proposed for the SST sub-array of CTA, designed and originally built by an Australian- British-Dutch-French-German-Japanese consortium. Because of their large number and the required lifetime of 30 years, one of the main design drivers for the SSTs should be to ease manufacturing, AIT (Assembly, Integration, and Test) and maintenance procedures and to limit their cost. GCT was designed according to these guidelines. Its optics are based on a Schwarzschild-Couder (SC) design which offers a large field of view (FoV), a good angular resolution for VHE over this entire FoV, a reduced focal length that decreases the physical pixel and camera sizes, allowing for a more compact and lightweight telescope structure and camera that are well adapted to new sensors such as silicon photomultipliers (SiPMs). This optical design consists of two reflectors and a curved focal surface inbetween, which provides good correction of aberrations at large field angles even for small focal ratios. The GCT structure will be equipped with a compact high-energy camera (CHEC). The GCT prototype (pGCT), installed at the Observatory of Paris on its Meudon site, recorded its first Cherenkov light on-sky in $2015^{[3,4]}$. Since then, the assessment of the performances of the pGCT telescope and cameras with regards to the CTA requirements is ongoing. Thanks to the return of experience from the AIT phase of the pGCT structure and a continuous RAMS (Reliability, Availability, Maintainability \& Safety) process, an optimised design of the telescope structure intended for the CTA Southern site was implemented in 2017-2018. The GCT consortium plans to install its first telescopes and cameras in Chile during a pre-production phase as soon as the site's infrastructure work is complete (including telescope foundations), the CTA Observatory final legal entity 
is in place and the In-Kind Contribution allocation process will be completed. Thereupon, the GCT consortium will contribute a certain number of telescopes to the subsequent CTA production phase. This paper provides an overview of the prototyping and development activities within the GCT consortium and describes the progress made on the GCT design for mass production since 2017.

\section{STATUS OF THE GCT PROTOTYPE}

Thanks to its SC optics, illustrated in Figure 2, the pGCT is lightweight (8.1 tons) and compact $(4.1 \mathrm{~m} \times 4.8 \mathrm{~m} \times 8.2 \mathrm{~m}$ in parking position). It is composed of four main subsystems which are the Mechanical Assembly, the Optical Assembly with a primary mirror of diameter D1 $=4 \mathrm{~m}$, a secondary mirror of diameter $\mathrm{D} 2=2 \mathrm{~m}$, a focal length of $\mathrm{F}=2.3 \mathrm{~m}$ and a focal ratio $\mathrm{f}=\mathrm{F} / \mathrm{D}=0.57$ - the Cherenkov Camera and Auxiliary Systems. The latter consists of the Telescope Control System (TCS): its hardware and software modules distributed between the slab cabinets, the cabinets embedded on the telescope and the PC server off-telescope; and a shelter. The small focal length of the telescope also implies that the $\sim 0.2^{\circ}$ angular pixel size required by CTA for the SSTs is achievable with pixels of physical dimensions of 6 to $7 \mathrm{~mm}$, while the dual-mirror optics ensures that the $80 \%$ containment diameter of the ideal point spread function (PSF) of the telescope is below $6 \mathrm{~mm}$ up to field angles of $4.5^{\circ}$. The required $\mathrm{FoV}$ of $8^{\circ}$ can therefore be covered with a camera of diameter $\sim 0.5 \mathrm{~m}$, composed of 2048 pixels. This allows the use of commercially available photosensors, significantly reducing the complexity and cost of the camera. In this section, the prototype telescope structure (Mechanical Assembly, Optical Assembly and Auxiliary Systems) is briefly recalled including design concept rationales. The progress made with the TCS implementation is presented, and the SiPM-based prototype camera (CHEC-S) is introduced along with laboratory test results.

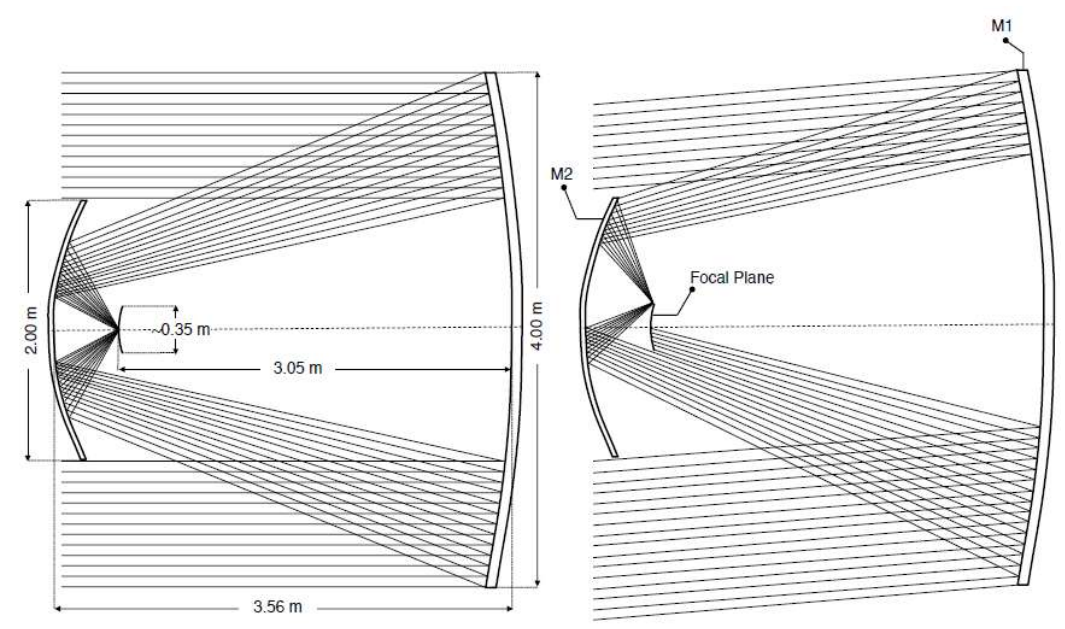

Figure 2. Optical layout of the GCT for the on-axis case (left) and at a field angle of $4.5^{\circ}$ (right) designed by J. Schmoll, Durham University. The primary and secondary mirror are labelled with "M1" and "M2", respectively.

\subsection{Technical design of the pGCT structure}

Figure 3 shows an annotated view of the GCT prototype with the major elements highlighted. The pGCT mechanical structure is mainly made of standard steel (E355) and contains three subsystems: (i) the Telescope Base which consists of a tower and its fixation to the foundation; (ii) the Altitude-Azimuth (alt-az) System (AAS) supported by the tower; (iii) the Optical Support Structure (OSS), which is attached to the AAS and holds the Optical Assembly. The primary mirror (M1) is segmented into six petals. The pGCT is currently equipped with two lightweight machined, polished and coated aluminium mirror segments, and with four dummies to simulate the mass and shape of the remaining segments. The secondary mirror (M2) is tessellated for manufacturing purposes, but its six high-precision segments are bolted together and behave like a monolithic mirror. The Optical Assembly and the primary dish support structure are separated to ensure that the stresses in the former do not directly influence the shape of the primary mirror segments. The Auxiliary Systems are composed of the TCS, which will be described in the next section, and a shelter. The shelter was installed in Meudon in 2014 to protect the pGCT (avoid excessive solar concentration risk, reduce ageing and degradation of the mirrors, enable maintenance operations in bad weather) and its installation in Chile is currently being considered by CTA. 
Early in the design development, the considerations of cost-effectiveness with regards to a future large-scale production and operations were taken into account. To this end, the M1 dish can be rotated along the telescope's optical axis to enable the installation and maintenance of mirror segments at ground level with the telescope in parking position. Subsequent locking of the support ensures the necessary stability. Moreover, the camera support includes a specific removal mechanism: a swivelling mount that provides easy access to the camera (lowered to a few centimetres above the ground). The system complexity at the level of the AAS is reduced by the use of similar designs for the azimuth and elevation motor shafts and drive systems. By simplifying integration and maintenance procedures, and therefore reducing their duration, the availability of the telescope on-site is increased. The use of commercial off-the shelf components and industrial standards ensures long-term availability and follow-up support.

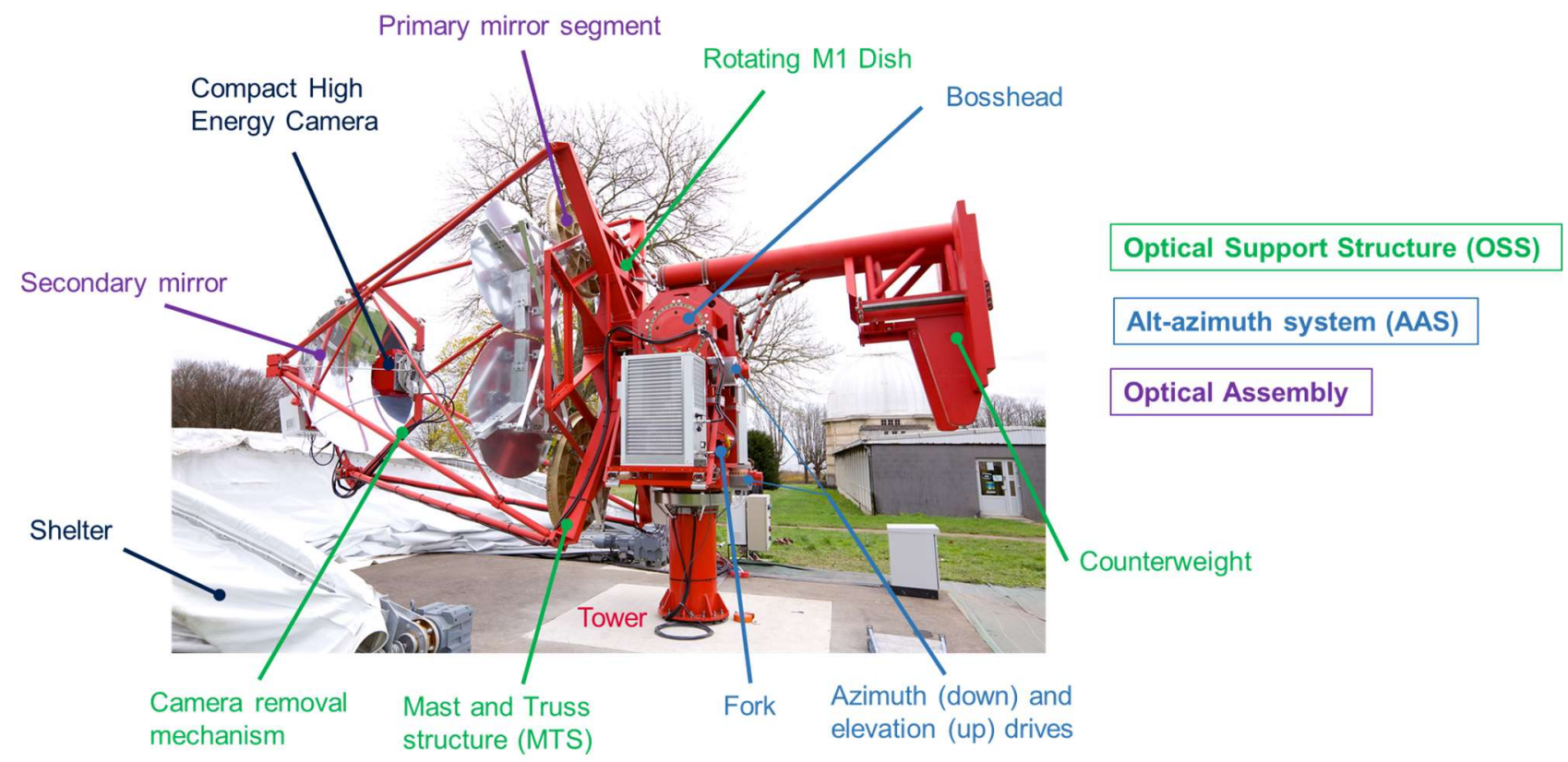

Figure 3. The pGCT installed in Meudon, with major elements indicated.

The verification of the design performances with respect to CTA requirements is ongoing. The mechanical structure has been fully validated ${ }^{[5,6]}$. The remaining work consists of consolidating the optical characterisation of the pGCT and software testing.

\subsection{Focus on the prototype Telescope Control System}

In the TCS of the pGCT the hardware core is an industrial PC (Beckhoff), as shown in Figure 4; it hosts the main Programmable Logic Controller (PLC SW) and the on-board control software.

The link for control and command to and from the distant devices is limited for the structure to a single OPC UA server. A NTP client/server provides the time synchronisation. The physical links are one Ethernet cable and the fibre connection in Figure 4, dedicated to the Cherenkov camera.

A single fieldbus (EtherCAT) is used for all distributed inputs/outputs. A dedicated hardware module (Beckhoff) hosts the safety PLC connected to all related sensors and actuators via the same fieldbus.

A proprietary network (TransnET) is required to manage the ETEL drives and torque motors in real-time via a PCI card (UltimET), set up and triggered in the Drive software and using the library provided by ETEL.

We present in the following sections the recent developments of the TCS. 


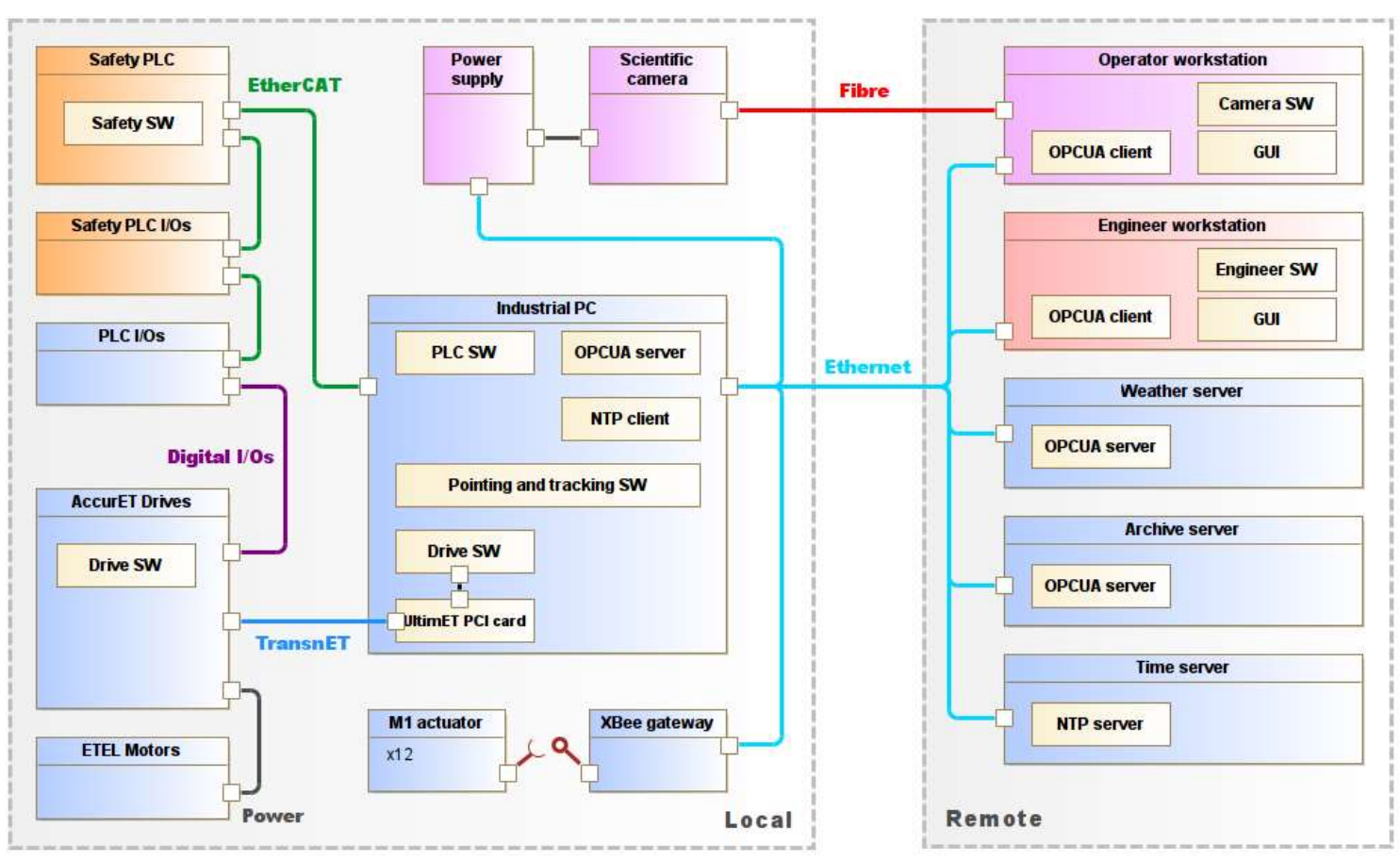

Figure 4. Simplified hardware and software architecture of the TCS from the telescope structure point of view (SW stands for software).

\section{State machine of the telescope structure}

Figure 5 shows the state machine managed by the main PLC. A brief description of the states is given in Table 1.

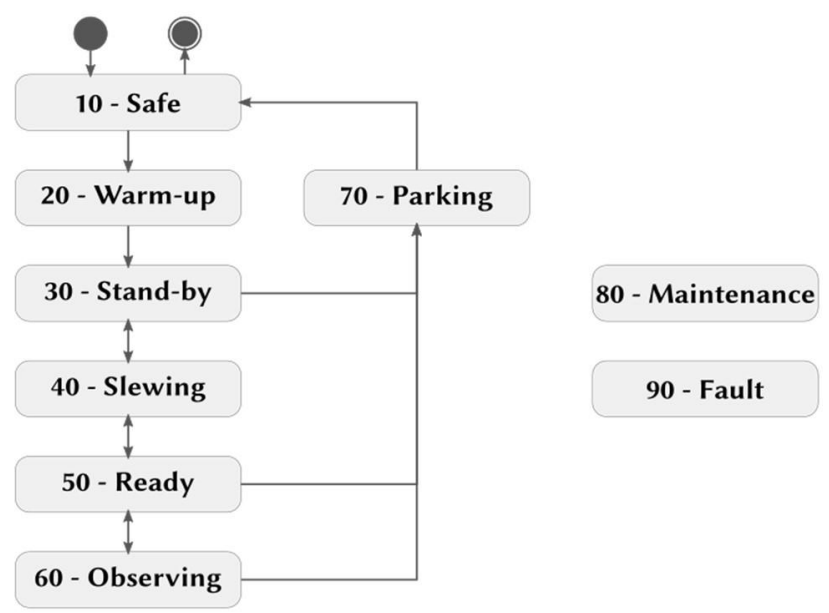

Figure 5. Simplified view of the state machine of the telescope structure. Note that the transitions to the states Maintenance and Fault are not shown due to their special use cases: the Fault state is activated upon failures and the access to the Maintenance state is restricted to the maintenance engineer. 
Table 1. Description of the states of the telescope structure.

\section{State Description}

\begin{tabular}{cl}
\hline Safe & State at power on and before power off - Motion is disabled. \\
\hline Warm-up & Transition state - Waiting for all devices to reach their operating status - Motion is disabled. \\
\hline Stand-by & The telescope structure is warmed-up but not pointing in the scientific area of the sky, above $20^{\circ}$. \\
\hline Slewing & Transition state - The telescope structure is in motion, towards a new pointing position. \\
\hline Ready & The telescope structure is ready to observe in the scientific area of the sky, above $20^{\circ}$. \\
\hline Observing & The telescope structure is performing a tracking to allow the scientific camera to observe the target. \\
\hline Parking & Transition state - The telescope structure is in motion, towards its parking position. \\
\hline Maintenance & State access is restricted to the maintenance engineer. \\
\hline
\end{tabular}

Some transitions are automatically executed, depending on the status of the telescope (moving, parked, warmed-up...) via the inputs of the main PLC, while others require the operator to send a command - e.g. an "enable" has to be transmitted to the main PLC to execute the transition between the Safe state and the Warm-up state.

\section{OPC UA protocol}

OPC UA is a publish-subscribe protocol: once a client has subscribed to a node managed by a server, any change on this node is notified along with the new value.

The OPC UA nodes of the TCS are of two kinds: 1) the nodes (of numeric, boolean or string type) reflecting measurements or states to monitor all the variables required to handle the telescope remotely (motor's temperature, power and power factor, strain gauges...); 2) the nodes used as gateways between the software modules. These nodes (of string type) are set as simplex communication gateways, e.g. the "EXT2PLC" node is dedicated to host the remote commands addressed to the main PLC and the "PLC2EXT" will host the acknowledgments sent back by the main PLC.

A new example of the use of these gateways, as seen in the preceding section, is the validation of transitions of the state machine.

\section{New setup of the drive control loop}

Previously, the motion of the pGCT was controlled with one motor shaft encoder per axis. This configuration required additional software development to compute the telescope position by counting the motor revolutions; moreover this position control method was degraded by a small backlash of the coupling imposing regular verifications of the pointing precision. To resolve these issues, a dual-loop feedback position control is now implemented in the TCS and is presented hereafter.

For each axis, azimuth and elevation, two rotary encoders (both from Heidenhain) are used: 1) an incremental one with 5000 periods per revolution and a $1 \mathrm{Vpp}$ sinusoidal interface is mounted on the motor shaft; 2) an absolute one with $2^{29}$ positions per revolution and an EnDat 22 interface is mounted on the slew bearing. The incremental encoder allows to drive the motor more precisely and the absolute encoder provides an improved position accuracy. The control loop now takes as measurement input both the actual telescope axis position and the motor shaft position rather than the latter alone (increasing its resolution). The pointing of the telescope is simpler and more accurate, but the control loop contains a gear with a transmission ratio of 1:114. Hence the values of the parameters of the proportional-integral-derivative controller (PID) have been completely revised.

The first slews with the new PID setup are satisfactory during the acceleration phase but showed an unwanted behaviour during deceleration due to the large inertia of the axis. With a small addition of the integral and derivative parameters, the movement of the azimuth axis is improved (see Figure 6). The tracking accuracy is enhanced compared to the previous setup (first tests); which was expected following the slewing tests since the accelerations and speeds during this kind of operation are very low. In addition, Figure 7 shows that the consumption remains low with the updated control loop. This configuration will be fully studied to consolidate the measurements. 

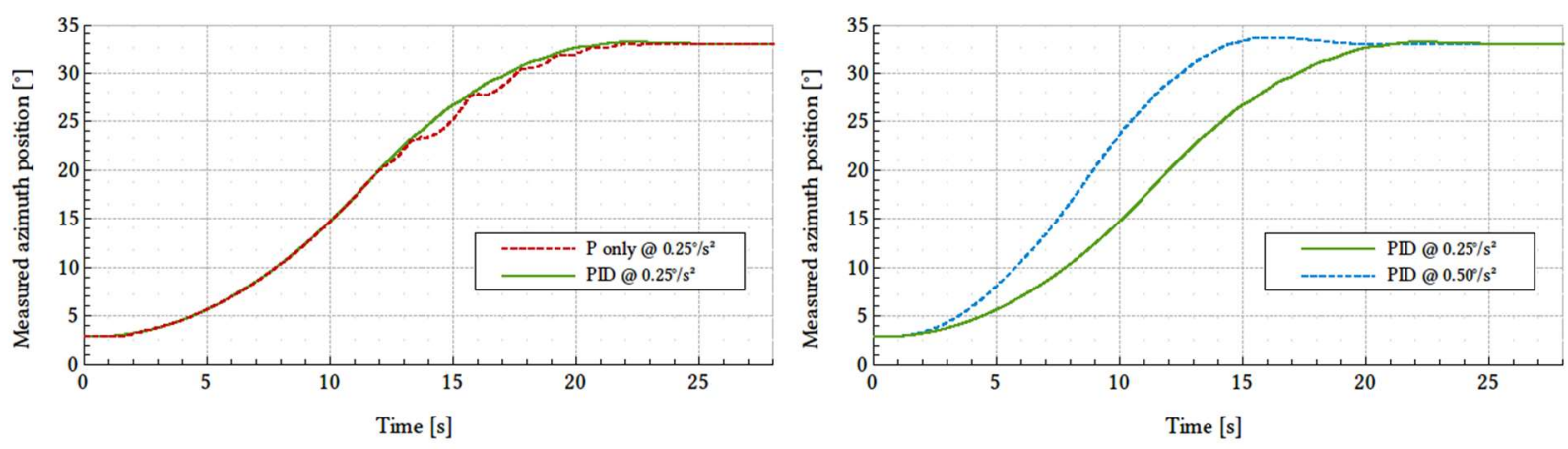

Figure 6. Motion example of the azimuth axis for two PID setups (left) and two maximum acceleration values (right). On the left, the raw setup curve (dashed red) shows small jolts in the deceleration phase. The first try setup (solid green) has removed most of this behaviour at the cost of a small overshoot. On the right, the same setup with a greater acceleration (dashed blue) again shows a good behaviour with a larger, but still acceptable, overshoot.

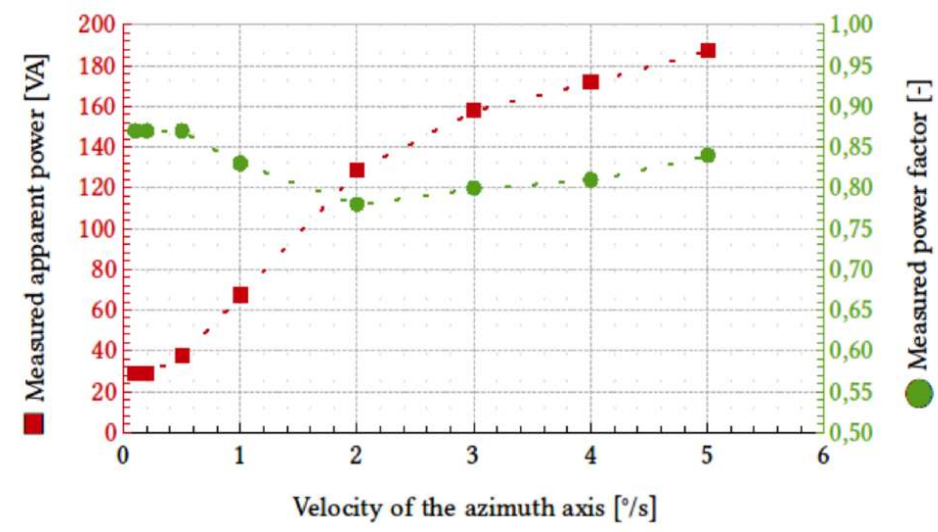

Figure 7. Measured apparent power (red) and power factor (green) of the azimuth axis while slewing with the new PID setup.

\section{Software of the pGCT}

The telescope software is distributed between (see Figure 4): the embedded Industrial PC (PLC SW, Drives $S W$ developed in the ETEL software environment ComET, Pointing and tracking $S W$ ), the Safety PLC (Safety SW), the drives controllers (Drives $S W$ written in $\mathrm{C} / \mathrm{C}++$ ) and, for the time being, the Engineer workstation in the control room (Active Mirror Control $S W)$. Interfacing with hardware is performed via the OPC UA communication protocol.

The automation software has been developed in accordance with the IEC 61131-3 standard in the TwinCAT environment. The following programming languages were used for the PLC SW and the Safety $S W$ : Structured text and Function Block Diagram.

The Pointing and tracking $S W$ has been developed in $\mathrm{C} / \mathrm{C}++$ and uses specific libraries for just-in-time multithread compilation and SOFA library for astronomical tools. The software architecture comprises 7 headers and 11 source files. It is responsible for the motors control, monitoring and parts of the safety (SW limit switches) as well as pointing, slewing and tracking taking into account the pointing and bending model corrections.

The interface to the standard communication protocol proposed for all CTA telescopes, based on the ALMA Common Software (ACS) developed to control the Atacama Large Millimetre Array (ALMA), will be developed in close cooperation with other CTA projects.

Automated setting of the M1 segments tip-tilt is carried out by Active Mirror Control (AMC) systems. A petal of the M1 mirror has been equipped with the prototype AMCs from Zürich University shown in Figure 8 (early B-series version, developed as a common component for CTA telescopes). These actuators are linked with a cord for power supply and use a wireless XBee protocol for control and command. The XBee gateway (ConnectPORT X2 from Digi) is embedded in the cabinet located behind the M1 mirror and connected to the local Ethernet network. 

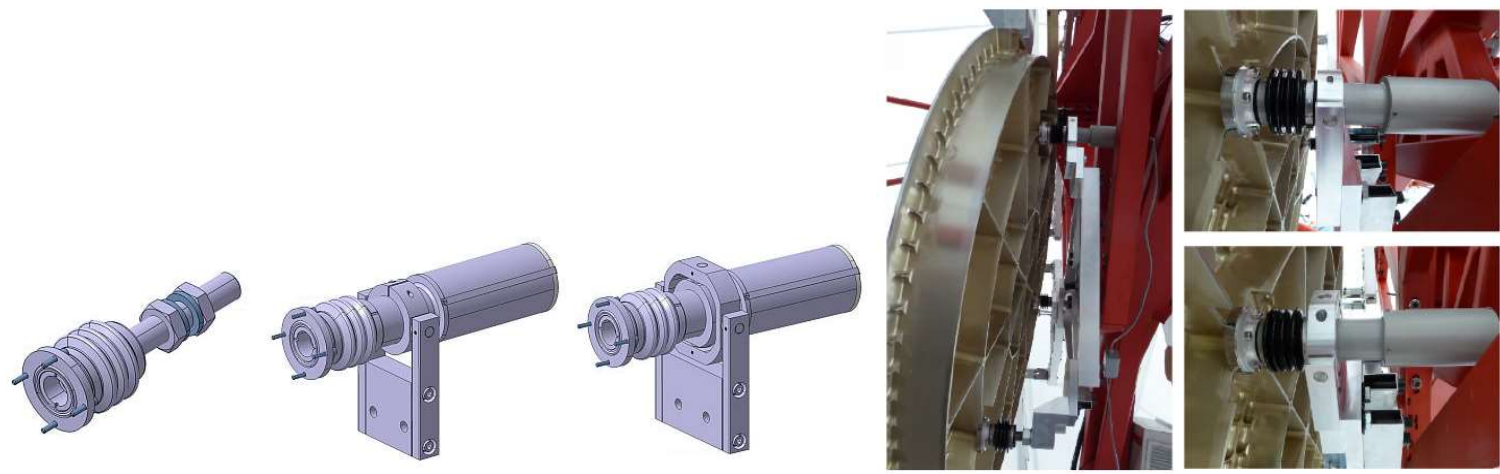

Figure 8. Left. Each M1 petal will be equipped with an AMC set, composed of: fixed point (left), 1-axis gimbal actuator (centre) and 2-axis gimbal actuator (right). The two actuators feature an XBee device to control their motor remotely. Right. Picture of the pGCT M1 segment equipped with AMC.

A server, developed in $\mathrm{C}++$, provides its clients with a connection with the actuators via the gateway. This server uses the libxbee library for the low-level exchanges and is hosted in the Engineer workstation in the control room. An additional piece of software, written in Python, allows the operator to remotely control the actuators or launch an automated process to align the petal. The latter is inspired by the methods used to align the mirror segments of the HESS telescopes ${ }^{[7]}$. Its implementation on the GCT prototype has been successfully validated on-sky. It has to be extended to address the 6 petals of the M1 before being commissioned, as well as its interface to the OPC UA server.

\subsection{The SiPM-based prototype camera}

The Compact High Energy Camera (CHEC) is the Cherenkov camera for GCT. CHEC consists of 2048 pixels grouped into 32 camera modules. Each module contains 64 pixels nominally $\sim 6 \times 6 \mathrm{~mm}^{2}$ in size and arranged in the focal plane to approximate the required radius of curvature resulting from the telescope optics. Internally camera modules connect to an intelligent backplane that provides camera triggering and routes data to data acquisition (DACQ) boards. The architecture of the CHEC internal elements is shown in Figure 9.

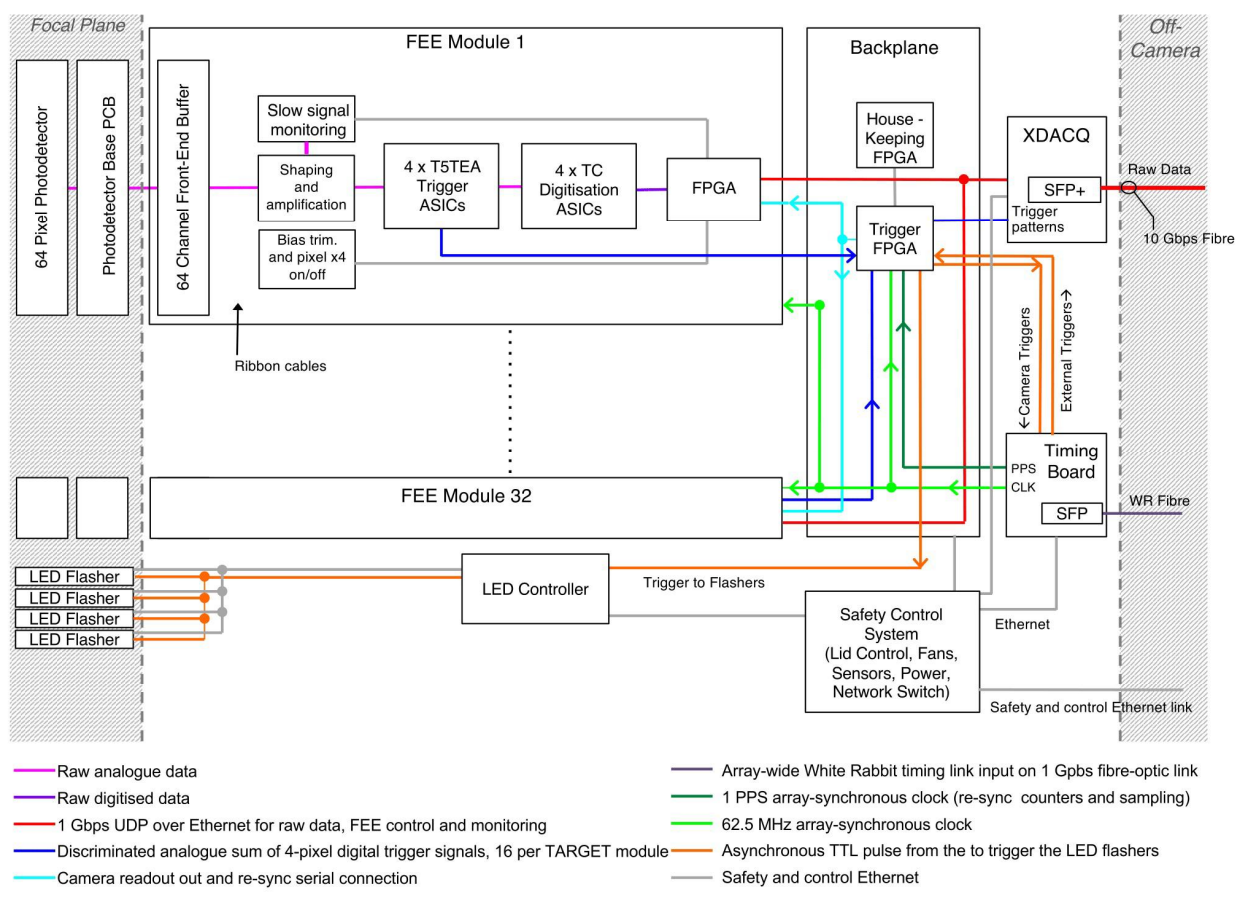

Figure 9. The CHEC architecture. 
The latest CHEC prototype (CHEC-S) features SiPMs. Each SiPM camera tile (Hamamatsu S12642-1616PA-50 - refer to ${ }^{[8]}$ for details) contains $2563 \times 3 \mathrm{~mm}^{2}$ pixels, combined in groups of four on a bias board directly mounted to the SiPM to provide the desired camera pixel size. A picture of CHEC-S is shown in Figure 10.

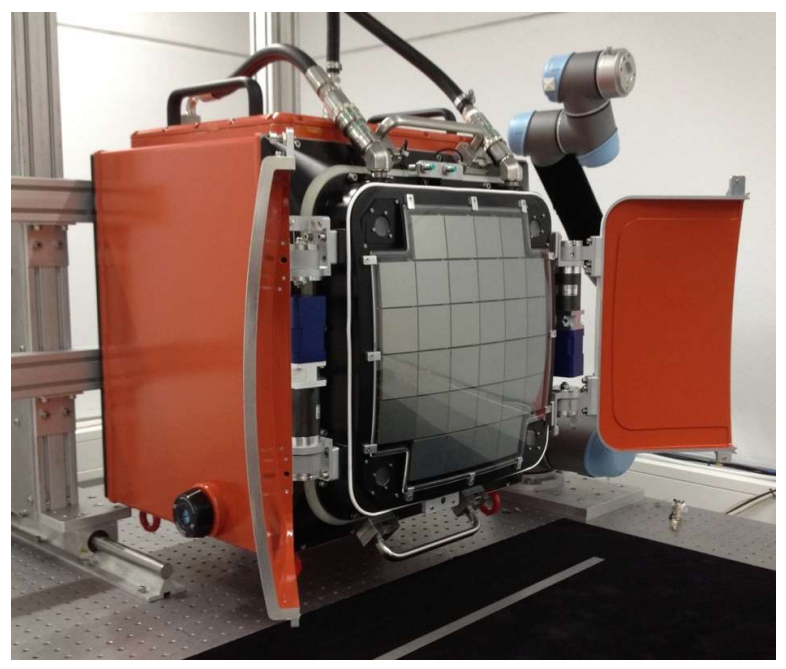

Figure 10. CHEC- $\mathrm{S}$ installed for lab tests.

Each SiPM tile is connected to a Front-End Electronics (FEE) module (see Figure 11) based on TARGET ASICs ${ }^{[9]}$ that provide waveform digitisation and a first-level camera trigger (implemented as a binary output from the analogue sum of four neighbouring pixels passing a threshold). The latest generation of TARGET ASICs are used in the CHEC-S FEE modules. Four 16-channel TARGET C ASICs provide sampling whilst four 16-channel T5TEA ASICs are used for triggering. The TARGET C ASIC is a 12-bit device that provides an effective dynamic range of 1 to $\sim 500$ photoelectrons for CHEC. The recovery of larger signals offline is possible due to the waveform digitisation. The sampling rate of TARGET C is tuneable, but nominally set to $1 \mathrm{GSa} / \mathrm{s}$ for CHEC. The size of the readout window digitised from the storage array is adjustable in 32 ns blocks set to 96-128 ns for CHEC to capture high-energy, off-axis events as they transit the focal plane. A slow-signal digitisation chain providing a per-pixel measurement of the DC light level in the photosensors is included in the FEE modules. This may be used to monitor the pointing of the telescope via stars during normal operation (see below). Module control and raw data output is via UDP over a 1 Gbps Ethernet link. An FPGA on-board each FEE module is used to configure the ASICs and other module components, to read-out raw data from the ASICs, and to package and buffer raw data for output from the module. Figure 12 shows average waveforms in CHEC-S from an increasing input light level (left) and an overlay of charge spectra from 2000 pixels of CHEC-S with an average illumination level of approximately 1 photoelectron (right).

The 32 camera FEE modules are connected to a backplane that provides the interface for power, clock, trigger and data. It forms a nanosecond-accurate camera trigger decision by combing signals from all FEE modules in a single FPGA. The FPGA used for triggering accepts all 512 first-level trigger lines from the FEE modules and implements a camera-level trigger algorithm (nominally set to require a coincidence between two neighbouring FEE trigger patches). Following a camera trigger, a serial message containing a unique event identifier is sent to the FEE modules to retrieve data from the sampling ASICs at the appropriate position in their memory. Data and communication links to the FEE modules are routed over the backplane to the DACQ board and then off-camera via fibre-optic link. Two prototype DACQ boards are used in CHEC-S providing four 1 Gbps links. In the final system a new DACQ board (referred to as the XDACQ board, and currently being tested) will provide a single $10 \mathrm{Gbps}$ to the camera. An array-wide White Rabbit system 110$]$ connected to a timing board inside the camera provides absolute timing.

Each corner of the camera is equipped with an LED flasher unit to provide calibration via reflection from the secondary mirror. The LED flasher units each contain ten LEDs, configured in a set of patterns to provide illumination over a wide range of intensities ${ }^{[11]}$. An external lid system provides protection from the elements. Thermal control of the camera is via an external chiller mounted on the telescope. Chilled liquid is circulated through the camera focal plane plate and a thermal exchange unit on the camera body. Six fans internal to the camera circulate the resulting cooled air. The camera is hermetically sealed and a breather-desiccator is used to maintain an acceptable level of humidity and atmospheric pressure within the instrument. 


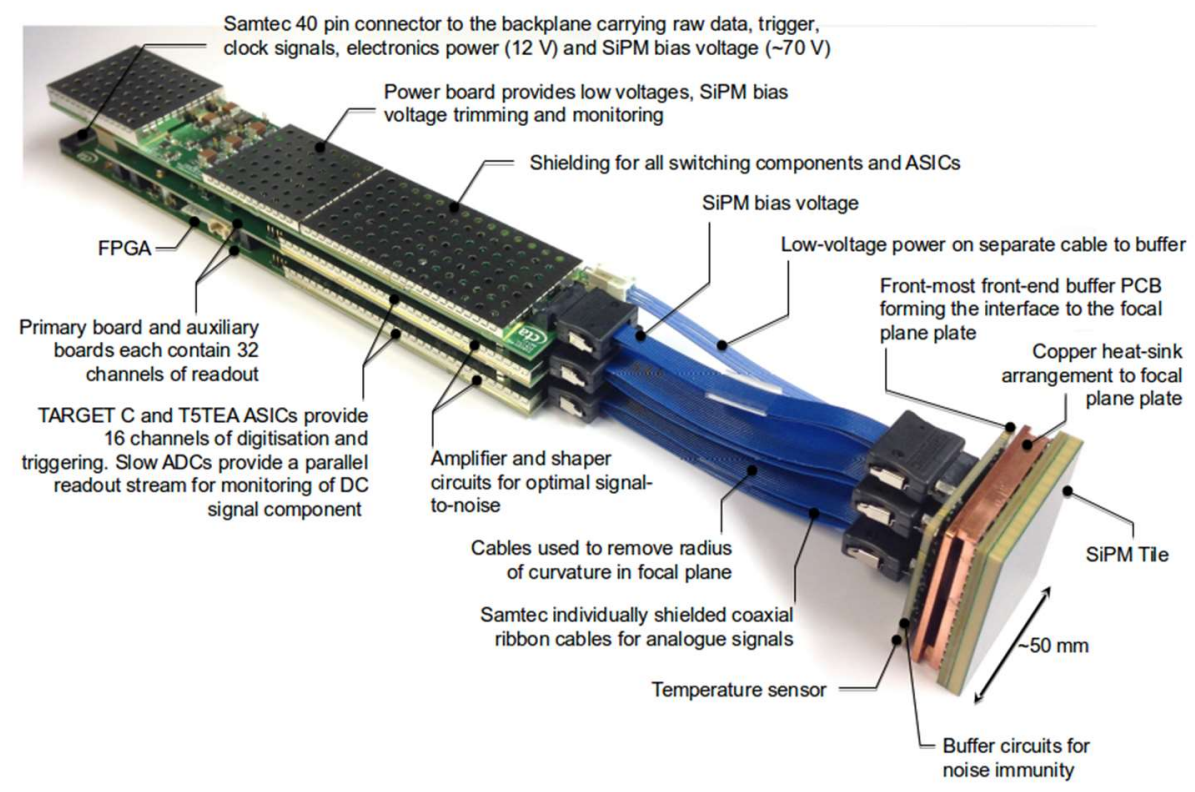

Figure 11. CHEC-S camera module.
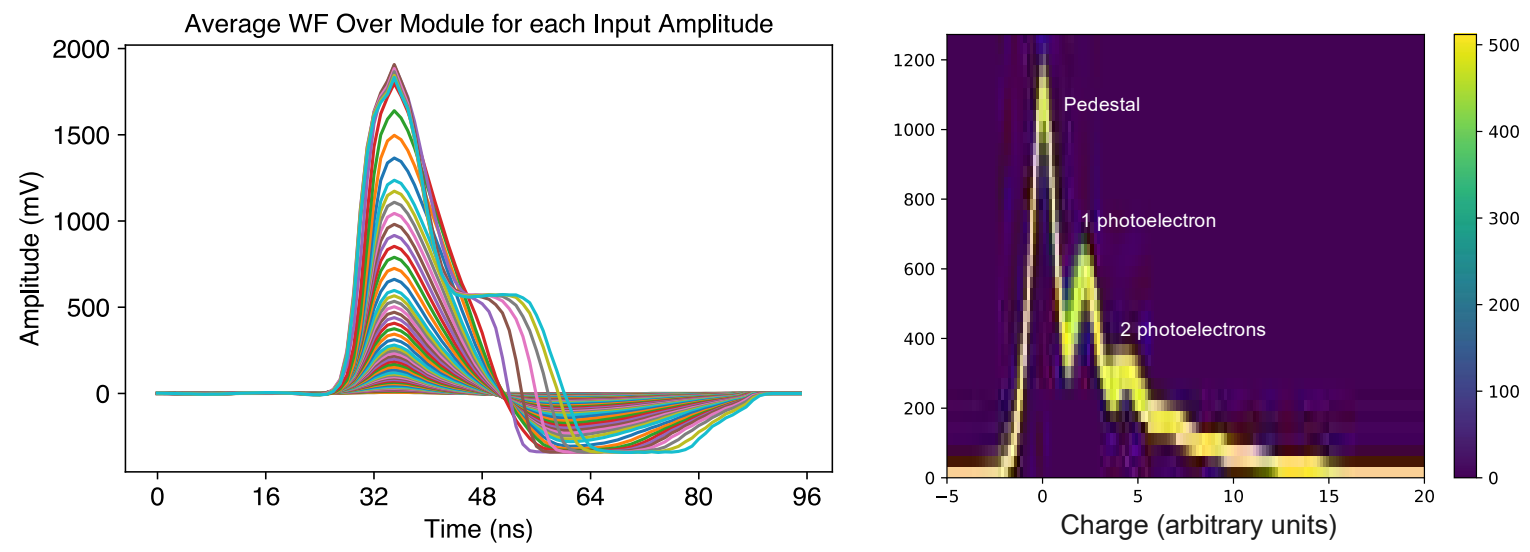

Figure 12. Average waveforms in CHEC-S from an increasing input light level (left) and an overlay of pulse-area spectra from $\sim 2000$ pixels of CHEC-S with an average illumination level of approximately 1 photoelectron (right).

The pointing monitoring system uses the DC offset of the Cherenkov camera to monitor the positions of (bright) stars in the FoV (slow-signal digitisation chain). The advantage of this implementation of telescope pointing offsets monitoring is that it measures directly the pointing of the camera, rather than trying to derive the pointing of the camera from orientations of various components in the telescope. Thus, no additional hardware is required, except for verification purposes, and it can be done continuously. During science data taking, the pointing system is by design running in parallel and able to deliver near-instantaneous pointing information. The main drawback of this method is that the Cherenkov camera is undersampling the PSF of the telescope, requiring high signal-to-noise measurement to achieve the required precision. To facilitate the pointing monitoring, the signal from the Cherenkov pixels is split in a fast channel (for the Cherenkov events) and a slow, quasi DC, channel that samples the DC offset at 10 millisecond resolution. Using correlation algorithms and edge detection schemes the star position on the Cherenkov camera can be determined to sub-pixel accuracy.

Calibration of the pointing monitoring system will be a two-step process. To provide first-order calibration and in order to validate the scheme, the Cherenkov camera is partly covered with a scattering screen that is monitored through a hole in the centre of the secondary mirror by a CCD camera. By using a scattering screen, a representative PSF is monitored in the high-numerical aperture beam of the telescope. A zoom lens on the CCD camera for monitoring the star images on the 
scattering screen allows for high-resolution sampling of the PSF of the telescope. By running parallel pointing observations on the Cherenkov camera in the open areas of the scattering screen and the external CCD camera, the correspondence between the Cherenkov pointing and the pointing obtained using the well-sampled PSF on the scattering screen can be established. A second calibration step is done on-the-fly using the Cherenkov camera itself. Pointing the telescope at a fixed point in the sky, the drifting of the stars can be used to calibrate the pixel response of the Cherenkov camera, leading to the required accuracy. Depending on the stability of the telescope, this calibration might need to be repeated under different elevation and temperature regimes.

\section{PRE-PRODUCTION DESIGN}

Following the prototyping phases of telescope structure and cameras, changes must be considered to adapt the design to mass production and new specific CTA constraints for the forthcoming pre-production phase. The inputs to this redesign are the experience and lessons learnt from the prototype's Assembly, Integration and Validation stages, the outputs of the RAMS processes and finally the wish to increase the commonalities between CTA telescopes. The revised GCT Product Breakdown Structure is introduced in Table 2.

Table 2. Pre-production GCT PBS.

$\begin{array}{lll}\text { ID } & \text { GCT PBS } & \text { 6G.2 } \\ \text { 6G.0 } & \text { Documentation } & \text { 6G.2.1 } \\ \text { 6G.0.1 } & \text { Project Management } & 6 \mathrm{G} .2 .2 \\ \text { 6G.0.2 } & \text { System Engineering } & 6 \mathrm{G} .2 .3 \\ \text { 6G.0.3 } & \text { Product Assurance } & \mathbf{6 G . 3} \\ \text { 6G.0.4 } & \text { Scientific documents } & 6 \mathrm{G} .3 .1 \\ \text { 6G.1 } & \text { Mechanical Assembly } & 6 \mathrm{G} .3 .2 \\ \text { 6G.01.01 } & \text { Telescope base } & 6 \mathrm{G} .3 .3 \\ \text { 6G.01.01.01 } & \text { Tower } & 6 \mathrm{G} .3 .4 \\ \text { 6G.01.02 } & \text { Optical Support Structure } & 6 \mathrm{G} .3 .5 \\ \text { 6G.01.02.01 } & \text { Mast and Truss Structure } & 6 \mathrm{G} .3 .6 \\ \text { 6G.01.02.02 } & \text { Camera Access } & \mathbf{6 G . 4} \\ \text { 6G.01.02.03 } & \text { Dish M1 } & 6 \mathrm{G} .4 .1 \\ \text { 6G.01.02.04 } & \text { Counterweight } & 6 \mathrm{G} .4 .2 \\ \text { 6G.01.03 } & \text { Alt-Azimuth System } & 6 \mathrm{G} .4 .3 \\ \text { 6G.01.03.01 } & \text { Alt-Azimuth structure } & 6 \mathrm{G} .4 .4 \\ \text { 6G.01.03.02 } & \text { Alt-Azimuth axes } & 6 \mathrm{G} .4 .5 \\ \text { 6G.01.03.03 } & \text { Mechanical supports for other assemblies } & 6 \mathrm{G} .4 .6 \\ & & 6 \mathrm{G} .4 .7 \\ & & 6 \mathrm{G} .4 .8\end{array}$

\author{
Optical Assembly \\ Primary Mirror Structure \\ Secondary Mirror Structure \\ Optical alignment systems
}

Cherenkov camera

Camera Mechanics

Photodetectors

Camera Electronics

Calibration System

Camera Auxiliary System

Camera Software

Telescope Control System

Slab Cabinet

Telescope Cabinets

External Cables

Automation Software

Safety Software

Integrated Control Software

Calibration

Pointing Control

The optimisation of the telescope structure and mirrors design is outlined hereafter. Finally, the upcoming prospects towards a camera design for the production phases of CTA are discussed.

\subsection{Telescope structure}

The mechanical assembly design of the telescope has been optimised at the Observatory of Paris design office (GEPI laboratory), as shown in Figure 13, as well as the appropriate section of the PBS (6G.1) to the LLRU (Lower LineReplaceable Unit) level. The GCT telescope structure (GCT-STR) manufacturing, assembly, mounting and testing on-site during pre-production, and production, will be carried out in industry by a prime contractor. A unique public tender process, and therefore contract in the format of a framework agreement, is planned for the construction of every GCT-STR for CTA. The manufacturing process will not change drastically, with the possible exception of using moulding for some parts rather than machining and welding, which can help to decrease production cost and rate. 

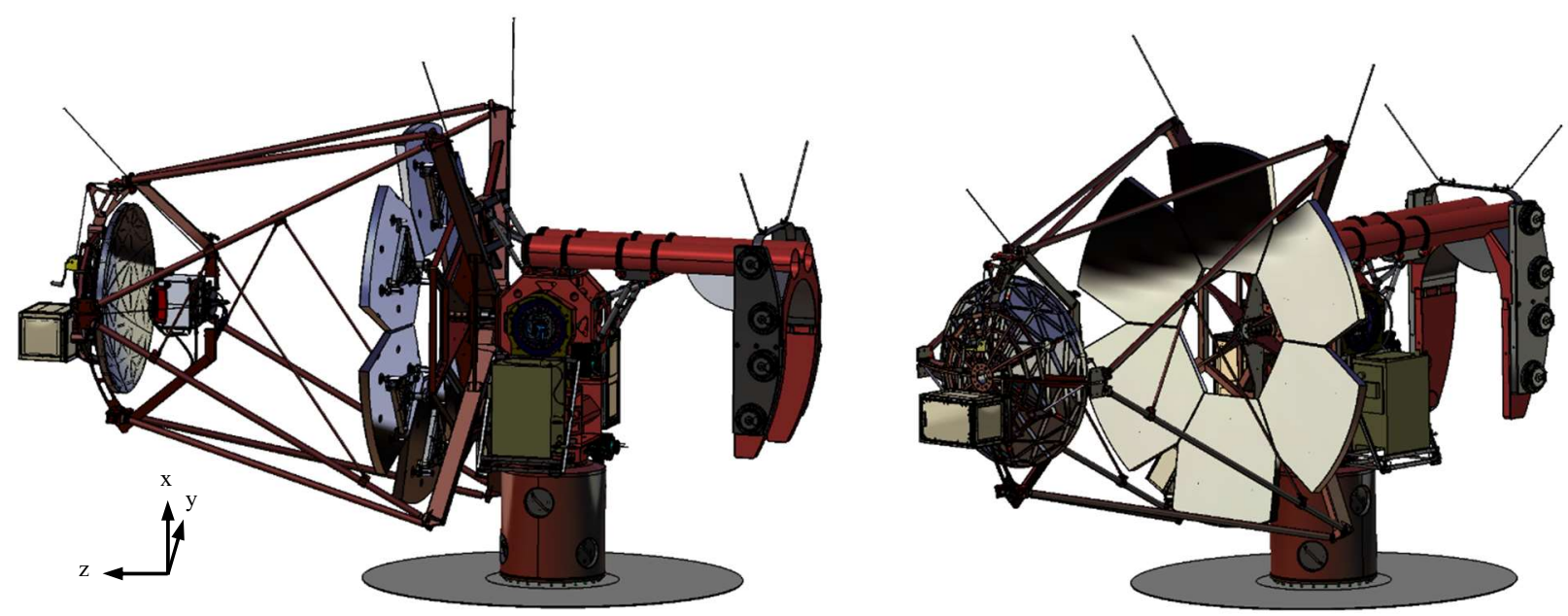

Figure 13. CAD model of the telescope design for pre-production implemented with CATIA v5 software. The camera integrated is CHEC-S.

The modifications implemented in the design are detailed below following the PBS.

The main modification driver concerning the tower (6G.01.01.01) was the willingness to develop a common design for the foundations of the three SST designs proposed for CTA. The tower diameter was increased to $1100 \mathrm{~mm}$ in order to have the same interface as the SST-1M telescope with the foundations, using 24 M30 threaded rods arranged on a ring of $1 \mathrm{~m}$ across. This change improves the stiffness of the tower and therefore its resistance to earthquakes. Moreover, the cables arriving through a manhole under or close to the tower will pass via its centre to the main cabinet while allowing the rotation of the telescope within a range of $520^{\circ}$ in azimuth. This implies that access holes and caps for installation of the cables in the tower must be foreseen and that the removal of the azimuth cable wrap is made possible (Figure 14). Those modifications impact the alt-azimuth structure and counterweight design.
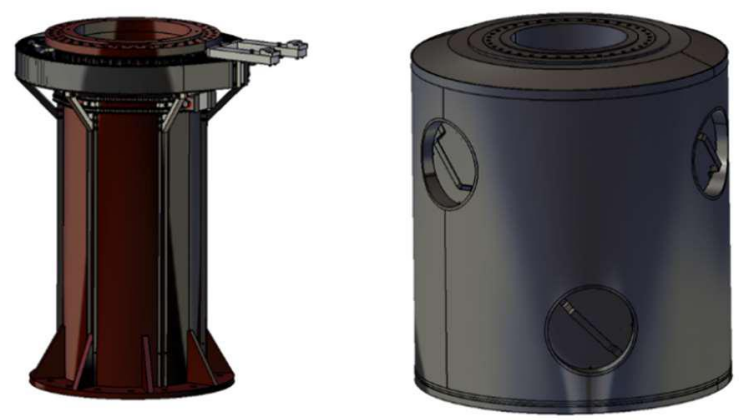

Figure 14. CAD views. On the left, pGCT tower with cable wrap. On the right, GCT pre-production tower design.

The dimensions of the fork and bosshead, the two sub-systems of the alt-azimuth structure (6G.01.03.01), were increased in order to ease the assembly of the fork. Moreover, some modifications were made to ensure the dust sealing of the motor shaft (IP65) and to ease maintenance (coupling of the motor to the shaft).

As for the prototype, we continue to use the same system (drives and motor shafts equipped with two motors) for azimuth and elevation. By cons, many parts of these sets, on GCT-STR, will be stainless steel.

The same drive system is used on both sides of the bosshead, one of them being motorized. In order to align the axes of rotation of the two systems, the elevation drive systems are placed on the fork via cylindrical soles. This interface system has been modified for GCT-STR to ease this assembly and to reduce the cost of mass production (see Figure 15). 

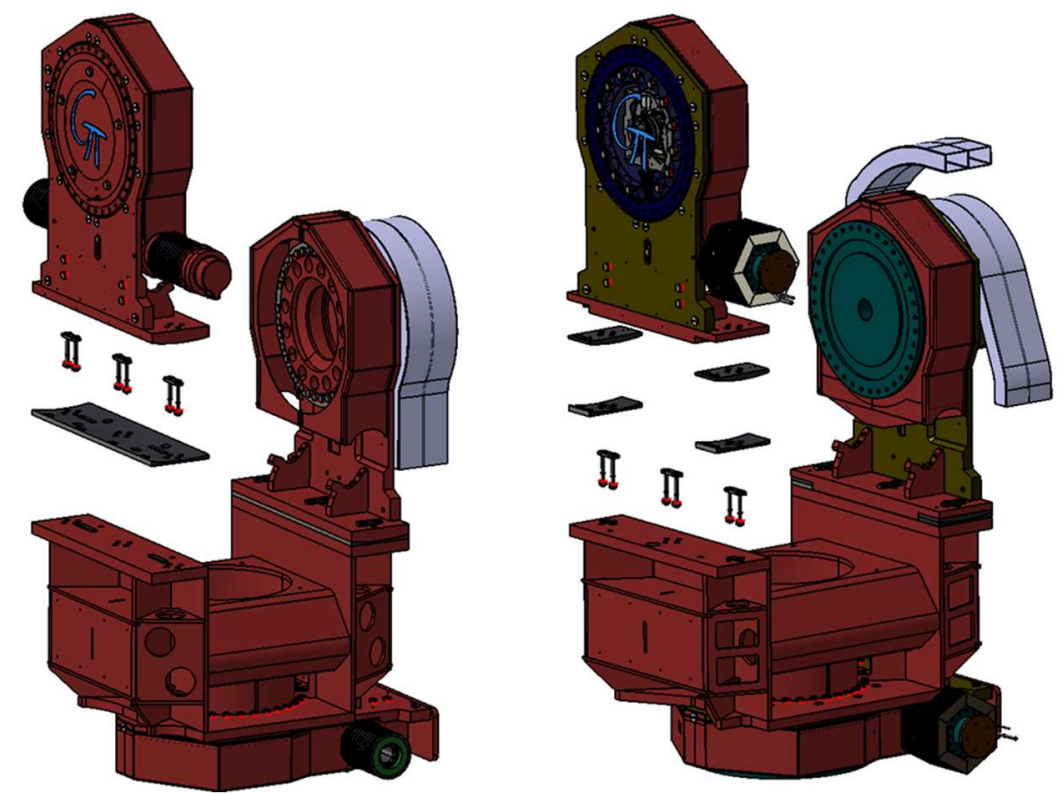

Figure 15. On the left, interface drive to fork on pGCT. On the right, interface drive to fork on GCT pre-production design.

The assembly of the motors on the shaft (Figure 16), for the drives of the alt-azimuth structure, have been optimised to reduce maintenance operations durations.

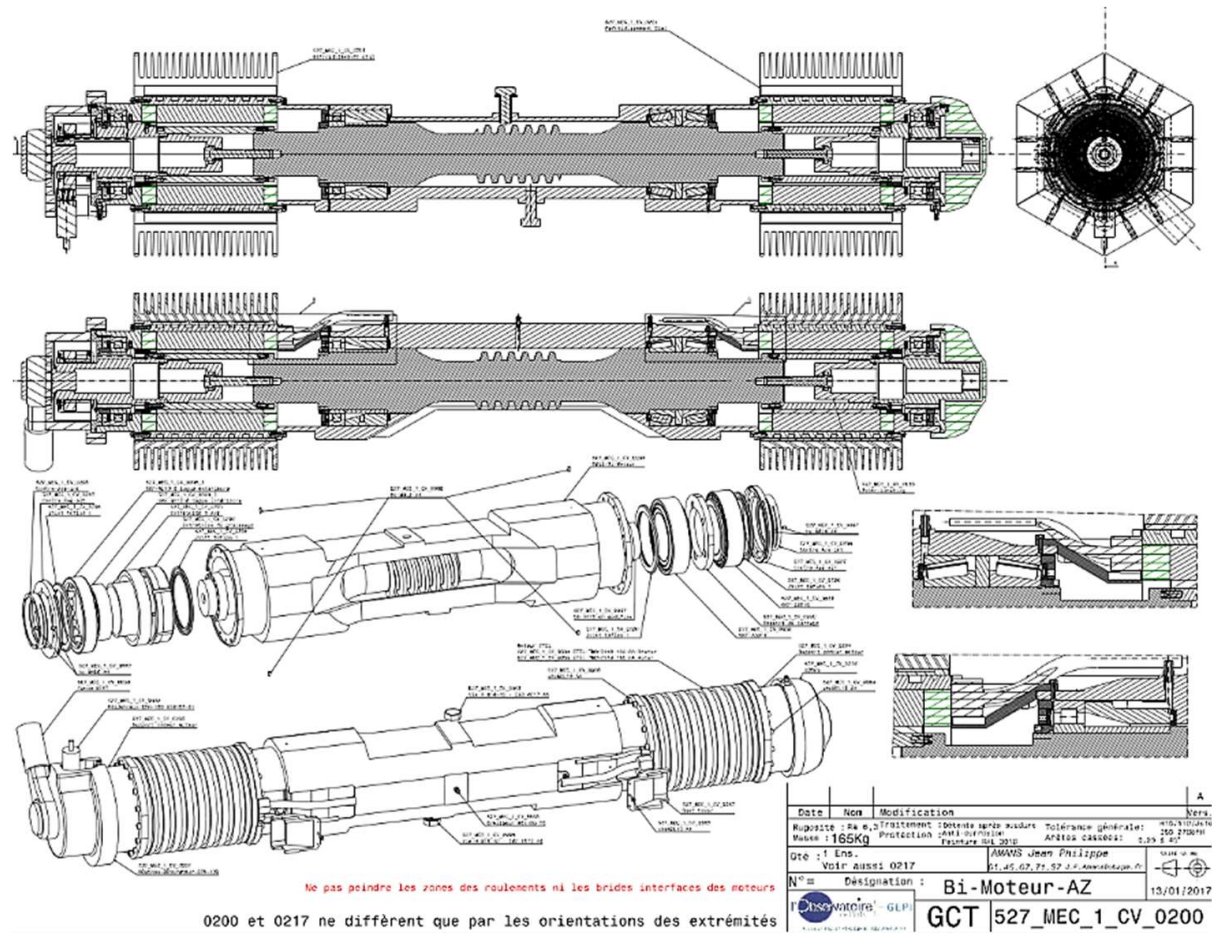

Figure 16. Blueprint of the motor shaft equipped with two motors.

The structure of the counterweight (6G.01.02.04) has been enlarged to allow the movement of the fixed mass system around the tower when the telescope points to the zenith (illustrated in Figure 17). This was implemented by increasing the space between the two main tubes. To enhance the resistance of the counterweight to CTA survival conditions (earthquakes and gusts of winds at maximum speed), the following changes were made: 
- The dimension of the fixing chains and their fastening to the mount were optimised.

- Interface beams to the Mast and Truss Structure and to the bosshead were reinforced by increasing their diameter and seismic reinforcements was added.

- $\quad$ The fixed mass shape was optimised.

Finally, the moving masses of the counterweight were removed and replaced by adjustment weights that can be added depending on the configuration of the telescope during maintenance operations (camera removed, mirrors removed, etc.).
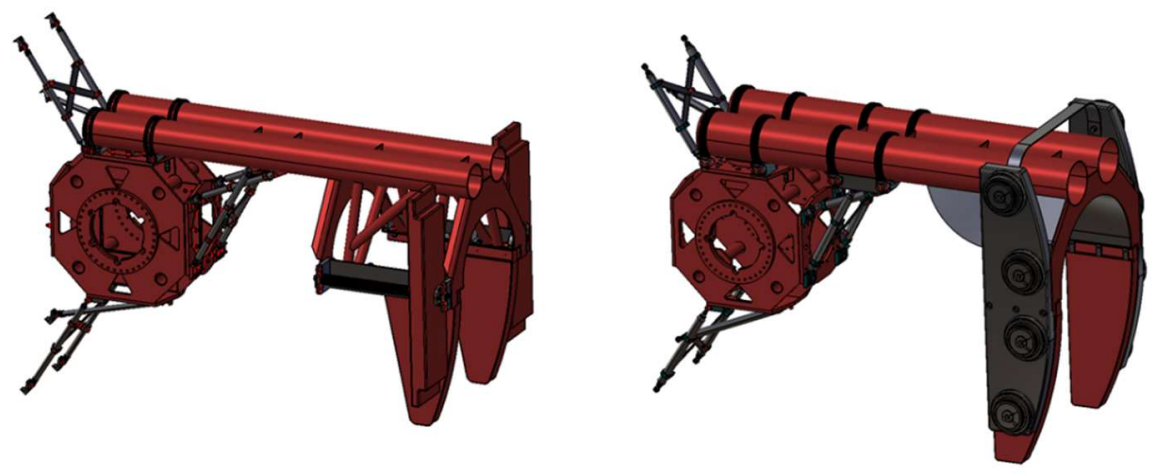

Figure 17. CAD views of the counterweight. On the left, the pGCT counterweight. On the right, the pre-production GCT counterweight with the circular adjustment weights.

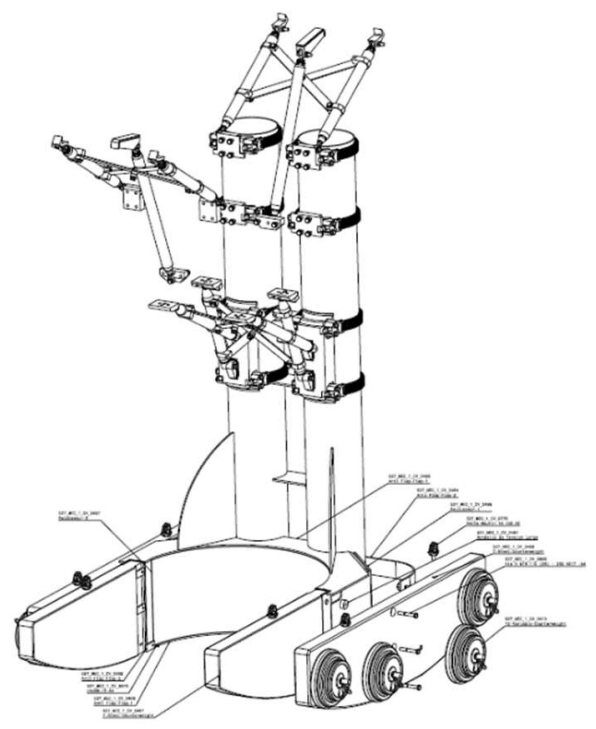

Figure 18. Extract of the pre-production GCT counterweight blueprint.

Regarding the Optical Support Structure (6G.01.02), one of the lessons learned from the pGCT was that additional degrees of freedom were useful in order to better align the optical elements (focus and decentring). To this end, the interface between the triangular supports of the M1 segments and the M1 dish (Figure 19) was modified to enable a translation along the $\mathrm{z}$ axis (parallel to the optical axis) thanks to an adjusting screw and guiding by a long hub. Additionally, adjustments of the position of the segment along the $\mathrm{x}$ and $\mathrm{y}$ axes are made possible by two perpendicular dovetails and pushing screws. For the tip-tilt alignment, motorised actuators developed by Zürich University as a common component for all CTA telescopes are mounted on the moulded triangular support structure. Note that the secondary mirror is the reference in the GCT optical scheme and will not be equipped with actuators. To adjust the centre of the camera focal plane (Figure 19) with regard to the optical axis, adjusting screws and guide rails are implemented on the support of the camera to provide the setting along the $\mathrm{x}$ and $\mathrm{y}$ axis. Furthermore, focus and tip-tilt alignment of the camera is allowed by three push-pull screws with $+/-11.5 \mathrm{~mm}$ range. 

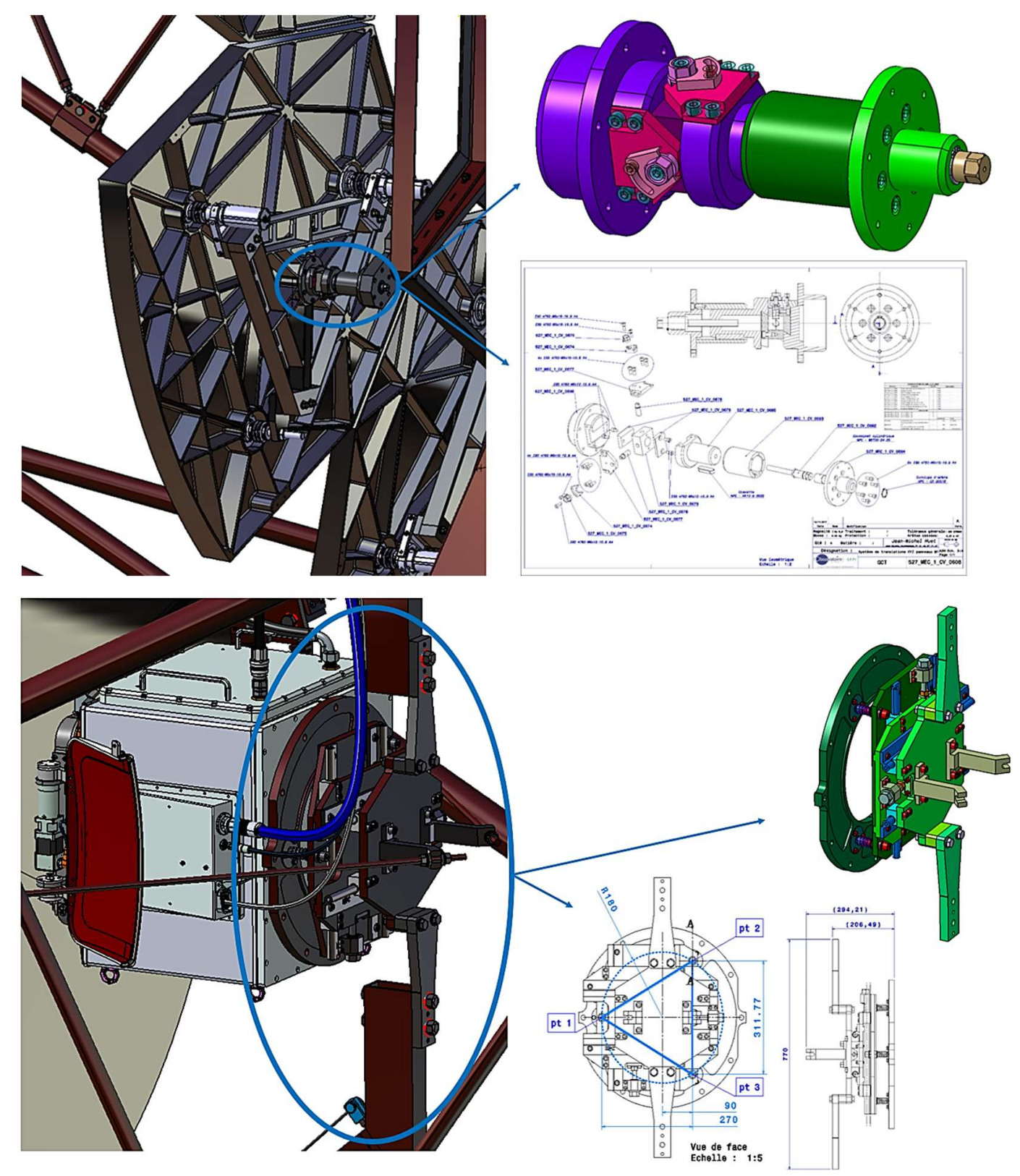

Figure 19. CAD views. Details of the interface between the mechanical structure and the M1 segments (upper panel) and of the camera positioning system (lower panel).

The optics-positioning budget is summarised in Table 3. The M2 is the reference in the optical scheme; we consider in this budget the optical elements that can be aligned therefore the camera and the M1 individual panels. For each of them the stroke and sensitivity of the positioning (given by the screw threads or actuator step) allowed by the mechanical elements described above are taken into account. The maximum mechanical errors (dimensional or angular) of an assembly is estimated as the sum of the uncertainties of each mechanical part (derived from ISO 2768 standards) and each interface (to take into account the errors due to the assembly). As the sum provides the most pessimistic value, a verification of the mechanical structure of the dish is envisaged. For the camera, the available play for decentring and defocus is oversized, since the mechanical errors estimated are inferior to the tolerances defined in the optical design, but will be useful for calibration purposes.

The air termination rods implemented at five positions of the structure, in compliance with the IEC 62305-3 standard (determined by a CTA wide study for lightning protection using the rolling sphere method with $20 \mathrm{~m}$ radius), increases the 
dimension of the telescope to $5.7 \mathrm{~m}$ in height (parking position). On the other hand, the top cabinet behind the secondary mirror is redimensioned to contain the camera Power Supply Unit and increases the telescope length to $8.5 \mathrm{~m}$. The GCT mass is around 10.8 tons (with the CHEC-S camera, camera chiller and telescope cabinets).

Table 3. Positioning budget of the M1 and camera in the GCT pre-production design. The balance is obtained by considering the total available stroke (\#1) and the tolerable amount of residual misalignment (2\#) subtracted by the maximum positioning error due to the manufacturing and assembly of mechanical parts (\#3). The M1 cannot be moved as a whole but is considered to verify that the stroke along the z-axis of individual panels is sufficient to compensate its mechanical error in focus but also the defocus caused by the tip-tilt alignment considering the individual panel and the global M1 (hence the grey areas where the effect is included in other parameters).

\begin{tabular}{|c|c|c|c|c|}
\hline & & M1 (global) & M1 (panel) & Camera \\
\hline \multirow{4}{*}{$\begin{array}{c}\# 1 \\
\text { Stroke }(+/-)\end{array}$} & $\mathrm{x}, \mathrm{y}$ & & $2.50 \mathrm{~mm}$ & $20.00 \mathrm{~mm}$ \\
\hline & $\mathrm{z}$ (focus) & & $12.50 \mathrm{~mm}$ & $7.00 \mathrm{~mm}$ \\
\hline & tip & & $89.40^{\prime}$ & $99.00^{\prime}$ \\
\hline & tilt & & $89.40^{\prime}$ & $114.60^{\prime}$ \\
\hline \multirow{4}{*}{$\begin{array}{c}\# 2 \\
\text { Sensitivity of the } \\
\text { setting }(+/-)\end{array}$} & $\mathrm{x}, \mathrm{y}$ & & $0.44 \mathrm{~mm}$ & $0.31 \mathrm{~mm}$ \\
\hline & $\mathrm{z}$ (focus) & & $0.44 \mathrm{~mm}$ & $0.44 \mathrm{~mm}$ \\
\hline & tip & & $0.10^{\prime}$ & $4.80^{\prime}$ \\
\hline & tilt & & $0.10^{\prime}$ & $5.40^{\prime}$ \\
\hline \multirow{4}{*}{$\begin{array}{c}\# 3 \\
\text { Optical design } \\
\text { tolerances }(+/-)\end{array}$} & $\mathrm{x}, \mathrm{y}$ & & $2.00 \mathrm{~mm}$ & $5.00 \mathrm{~mm}$ \\
\hline & $z$ (focus) & $5.00 \mathrm{~mm}$ & & $5.00 \mathrm{~mm}$ \\
\hline & tip & & $1.00^{\prime}$ & $8.40^{\prime}$ \\
\hline & tilt & & $1.00^{\prime}$ & $8.40^{\prime}$ \\
\hline \multirow{4}{*}{$\begin{array}{c}\text { \#4 } \\
\text { Mechanical } \\
\text { errors }(+/-)\end{array}$} & $\mathrm{x}, \mathrm{y}$ & & $3.55 \mathrm{~mm}$ & $1.95 \mathrm{~mm}$ \\
\hline & $\mathrm{z}$ (focus) & $10.50 \mathrm{~mm}$ & $3.55 \mathrm{~mm}$ & $2.35 \mathrm{~mm}$ \\
\hline & tip & & $10.80^{\prime}$ & $10.20^{\prime}$ \\
\hline & tilt & & $10.80^{\prime}$ & $10.20^{\prime}$ \\
\hline \multirow{4}{*}{$\begin{array}{c}\# 5 \\
\text { Positioning } \\
\text { budget balance }\end{array}$} & $\mathrm{x}, \mathrm{y}$ & & $>0$ & $>0$ \\
\hline & $z$ (focus) & $>0$ & & $>0$ \\
\hline & tip & & $>0$ & $>0$ \\
\hline & tilt & & $>0$ & $>0$ \\
\hline
\end{tabular}

\subsection{Mirrors}

The GCT optical design remains unchanged [12]; only the geometry of the M1 segments is modified with respect to the prototype to increase the M1 area. The mirrors were redesigned to achieve the maximum structural rigidity using topography optimisation techniques. The finite-element model with mesh of the pre-production M1 panel is shown in Figure 20 and presented in more details in these proceedings ${ }^{[13]}$.

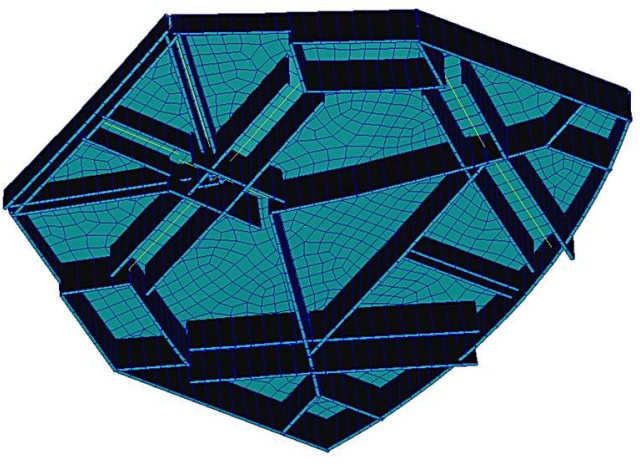

Figure 20. FEM of the intended primary panel for the pre-production telescope made with MD.Nastran. 
For cost-effectiveness, the mirror manufacturing process intended for pre-production, and production, is die casting. To achieve the required quality of the reflective surface (Table 4) the process consists of multiple steps: firstly, the aluminium alloy is casted (low-pressure) and machined to reach an intermediate roughness. Then, a protective layer (Nickel electroplating) is applied to improve the surface quality. The Nickel deposit also increases the service life of the panel by protecting it from corrosion. The thickness of this layer is based on the off-shore know-how to provide a life time longer than 30 years. The panel is then polished before applying the reflective coating (typically $\mathrm{Al}+\mathrm{SiO}_{2}+\mathrm{HfO}_{2}+\mathrm{SiO}_{2}$ ). In order to qualify this process, two pGCT-shaped panels are currently being manufactured following the previously mentioned steps, along with "witness samples" that will be used for surface characterisation. The results will be published at a later stage.

Table 4. Manufacturing specifications for the reflective surface of the M1. The high frequency deviations of the surface that induces scattering has seen its tolerance tightened compared to the first two pGCT M1 panels based on the experience acquired with Cherenkov telescopes $\frac{[14]}{\text {. }}$

\section{Error}

Tolerance

\begin{tabular}{|c|c|}
\hline $\begin{array}{l}\text { Surface waviness } \\
\text { (low spatial frequency deviations responsible for optical } \\
\text { aberrations related to Zernike's coefficients 2-21) }\end{array}$ & $20 \mu \mathrm{m}$ RMS \\
\hline $\begin{array}{l}\text { Surface irregularity } \\
\text { (high spatial frequency deviations responsible for optical } \\
\text { aberrations related to Zernike's coefficients 22-231) }\end{array}$ & $1 \mu \mathrm{m}$ RMS \\
\hline Micro-roughness & $7 \mathrm{~nm}$ RMS \\
\hline
\end{tabular}

The optical performances of the pre-production design were assessed using ROBAST (ROOT-based simulator for ray tracing), a non-sequential ray-tracing simulation library developed for wide use in optical simulations of gamma-ray and cosmic-ray telescopes ${ }^{[15]}$. The previous simulation model ${ }^{[16]}$ was updated to the pre-production design by taking into account the modifications of the structure and primary mirror contours. Simulations of the ideal optical performances of the telescope were carried out to determine the effective collection area and the shadowing induced by the secondary mirror, masts, trusses and camera housing as shown in Figure 21.

The shadowing of the telescope structure and camera results in a decrease of the collection area by $\sim 1 \mathrm{~m}^{2}$ for on-axis observations and $\sim 1.5 \mathrm{~m}^{2}$ towards the edge of the FoV. Later on, ROBAST will be used as an intermediate step to feed inputs to the CTA Monte Carlo tool (sim_telarray software) by reproducing the measured PSF of the prototype and extrapolating the optimised PSF of the pre-production design (comprising additional degrees of freedom to correct the misalignment of the optics).
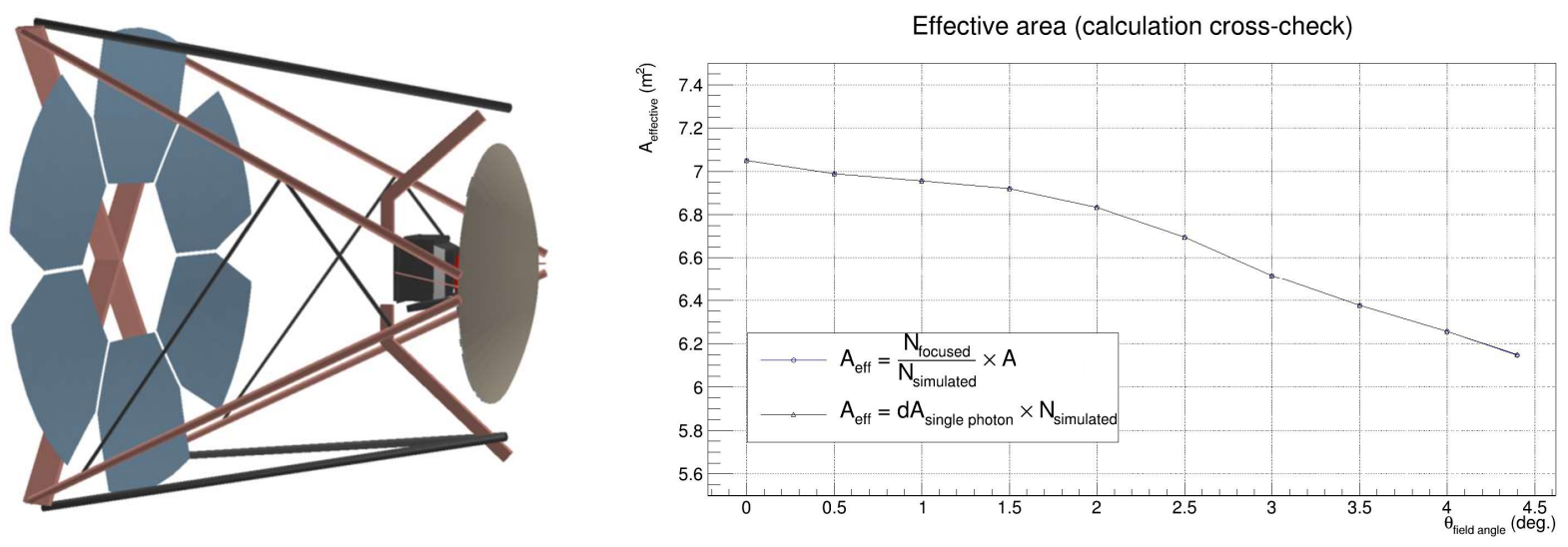

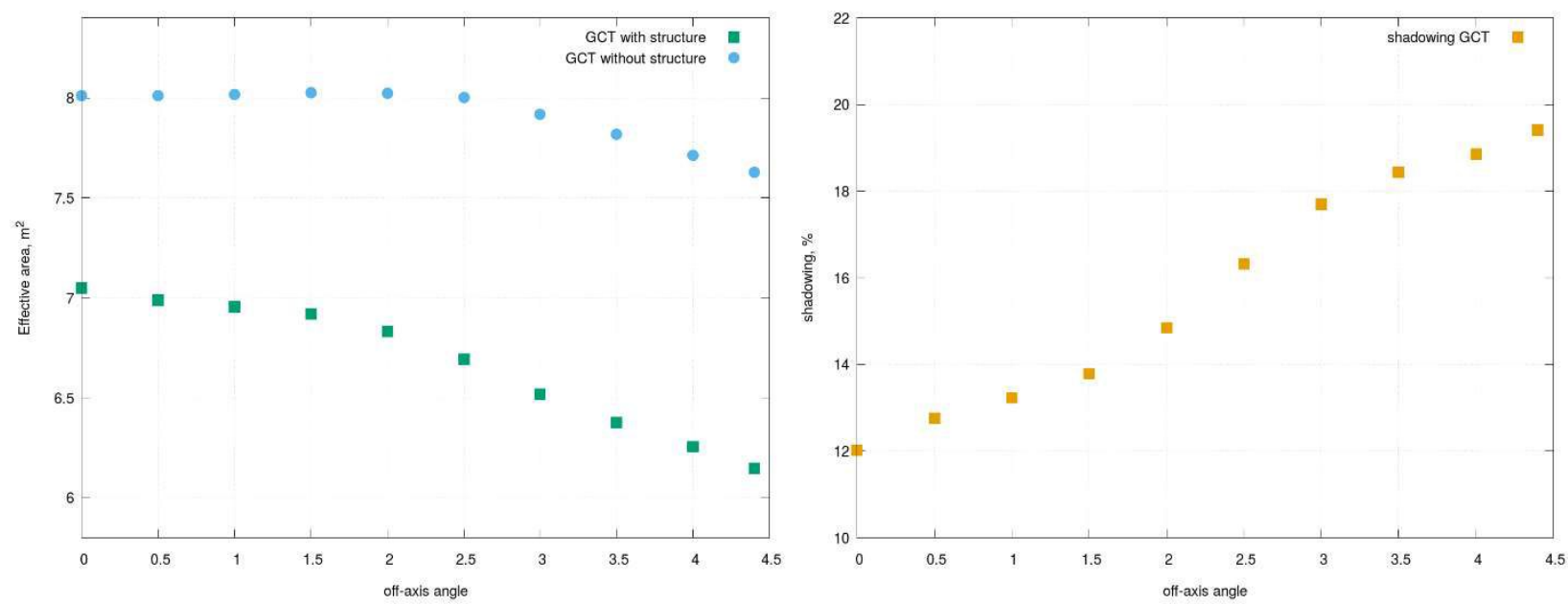

Figure 21. Top left: Computational model of GCT pre-production design comprising the segmented primary mirror, the monolithic secondary mirror, the Optical Support Structure masts and trusses and the camera housing, which includes an ideal focal surface (red). Top right: Effective collection area as a function of field angle given by $A_{\text {eff }}=\frac{N_{\text {focused }}}{N_{\text {simulated }}} \times A$ where $\mathrm{N}$ is the number of photons and A the M1 area. Bottom left: Difference between the effective collection area with shadowing components (green) and without (blue). Bottom right: The effect of shadowing of the telescope structure and camera is shown as a function of field angle. These simulations are valid for a single GCT and do not include photon detection efficiency or mirror reflectance.

\subsection{Beyond CHEC-S}

Plans are underway following prototyping to build, test and deploy three pre-production CHEC cameras on the SouthernHemisphere CTA site. Beyond this, we aim to provide cameras for a significant fraction of the 70 baseline SSTs during the production phase of CTA. With the notable exception of the SiPMs it is expected that the majority of components used in CHEC-S will also be used in the final production design of CHEC. SiPM technology is rapidly evolving and the latest devices offer significant performance improvements, including increased photodetection efficiency, lower optical cross talk and a reduced dependency of gain on temperature ${ }^{[17,18]}$. The camera nominally uses $20486 \mathrm{~mm}$ pixels. The use of slightly larger pixels up to $7 \mathrm{~mm}$ may result in improved performance due to the enlarged field of view despite an increase in night sky background, cost (which both scale as pixel area) and reduced angular resolution. To explore this trade-off, laboratory tests of the latest SiPMs and simulations with different pixel sizes are ongoing, with the aim of choosing a photosensor for the pre-production CHEC cameras in the coming months.

\section{SUMMARY AND OUTLOOK}

The pGCT installed at the Observatory of Paris on the Meudon site has been the first SST operated, since 2015, and controlled remotely since 2017. The final validation of the structure is scheduled before the end of the year, provided that the CTA requirements review is completed in a timely manner, including additional tests of several key-issues (PSF characterisation after final alignment and optimisation, performances of new moulded aluminium mirrors, pointing accuracy after updated TPOINT model of the telescope ${ }^{[19]}$ ). The CHEC-S AIV phase is progressing rapidly and two onsky campaigns are foreseen.

Using the experience and test results of the prototyping phase and industrial input on mass production processes, the mechanical structure of the pre-production telescope has been developed at the Observatory of Paris. The final design of the TCS and mirrors aims to be frozen by the end of the year. Tenders will be issued for the GCT structure manufacturing and AIV on-site after the CTA simplification process (work is in progress to increase commonalities between the different SST telescopes and cameras) and completion of the CTA Critical Design Review. Elements of the cameras will be constructed in industry, for example printed circuit boards and mechanical enclosures, but assembly, integration and performance verification will be carried out by GCT institutes. The pre-production phase will be used to ensure that a sufficient number of institutes develop camera integration and testing expertise to allow the production run that follows to be completed in a timely manner: current plans foresee three assembly centres. 


\section{ACKNOWLEDGMENTS}

This work was conducted in the context of the CTA Consortium. We gratefully acknowledge support from the agencies and organizations listed under Funding Agencies at this website:

http://www.cta-observatory.org/consortium_acknowledgments

\section{REFERENCES}

[1] Acharya, B. et al., "Introducing the CTA concept," Astroparticle Physics 43, 3-18, (2013)

[2] Montaruli, T., Greenshaw, T., Sol, H., Pareschi, G., For the CTA consortium and the SST-1M, GCT and ASTRI sub-consortia, "The small size telescope projects for the Cherenkov Telescope Array," Proceedings of the 34st ICRC, (2015)

[3] Dournaux, J.L., et al, The GCT consortium, "Operating performance of the gamma-ray Cherenkov telescope: An end-to-end Schwarzschild-Couder telescope prototype for the Cherenkov Telescope Array," Proceedings of the Vienna conference on instrumentation 2016, Nuclear instruments and methods in Physics research section A, 845, $355-358,(2017)$

[4] Watson, J.J., et al., For the CTA Consortium, "Inauguration and First Light of the GCT-M Prototype for the Cherenkov Telescope Array," Proceedings of the 6th International Symposium on High-Energy Gamma-Ray Astronomy, (2016)

[5] Dournaux, J.L., et al., For the CTA Consortium, "Performance of the Gamma-ray Cherenkov Telescope structure, a dual-mirror telescope prototype for the future Cherenkov Telescope Array," Proceedings of SPIE 9912, (2016)

[6] Le Blanc, O. et al, For the CTA GCT project, "Towards final characterization and performance of the GCT prototype telescope structure," Proceedings of the 35th International Cosmic Ray Conference, (2017)

[7] Cornils, R. et al., "The optical system of the H.E.S.S. imaging atmospheric Cherenkov telescopes, Part II: mirror alignment and point spread function,” Astroparticle Physics 20, 129-143, (2013)

[8] White, R., Schoorlemmer, H., For the CTA GCT Project, "A Compact High Energy Camera (CHEC) for the Gamma-ray Cherenkov Telescope of the Cherenkov Telescope Array," Proceedings of the 35th International Cosmic Ray Conference, (2017)

[9] Funk, S. et. al., "TARGET: A Digitizing And Trigger ASIC For The Cherenkov Telescope Array," Proceedings of the 6th International Symposium on High-Energy Gamma-Ray Astronomy, (2016)

[10] Serrano, J. et. al., "The White Rabbit Project,” Proceedings of ICALEPCS TUC004, Kobe, Japan, (2009)

[11] Brown, A.M. et. al., "Flasher and muon-based calibration of the GCT telescopes proposed for the Cherenkov Telescope Array," Proceedings of the 34th International Cosmic Ray Conference, Vol. POS(ICRC2015), (2015)

[12] Dournaux, J.L., et al., For the CTA Consortium, "Aspherical mirrors for the Gamma-ray Cherenkov Telescope, a prototype for the future Cherenkov Telescope Array," Proceedings of SPIE 9912, (2016)

[13] Dournaux, J.L., et al., For the CTA GCT Project, "Application of topography optimization techniques to the design of a lightweight primary mirror for the GCT, a dual-mirror telescope for the Cherenkov Telescope Array," in these proceedings (Conference 10706)

[14] Tayabaly, K., et al., "Roughness tolerances for Cherenkov telescope mirrors," Proceedings of SPIE 9603, (2015)

[15] Okumura, A., et al., "ROBAST: Development of a ROOT-Based Ray-Tracing Library for Cosmic-Ray Telescopes and its Applications in the Cherenkov Telescope Array," Astroparticle Physics 76, 38-47 (2016)

[16] Rulten, C., et al., "Simulating the optical performance of a small-sized telescope with secondary optics for the Cherenkov Telescope Array," Astroparticle Physics 82, 36-48 (2016)

[17] Tajima, H. et. al., "Evaluation of Silicon Photomultipliers for the GCT and ASTRI cameras of CTA," Proceedings of the 8th International on New Developments in Photodetection, (2017) 
[18] Otte, A.N. et. al., "Characterization of three high efficiency and blue sensitive silicon photomultipliers," Nuclear Instruments, Methods A, Vol. 846, p. 106, (2017)

[19] P. Wallace, “A rigorous algorithm for telescope pointing,” Proceedings of SPIE 4848, (2002)

Proc. of SPIE Vol. 10700 1070010-20

Downloaded From: https://www.spiedigitallibrary.org/conference-proceedings-of-spie on 1/15/2019 Terms of Use: https://www.spiedigitallibrary.org/terms-of-use 